\title{
Extensive Security Verification of the LoRaWAN Key-Establishment: Insecurities \& Patches
}

\author{
Stephan Wesemeyer ${ }^{1}$, Ioana Boureanu ${ }^{1}$, Zach Smith ${ }^{2}$, Helen Treharne ${ }^{1}$ \\ ${ }^{1}$ University of Surrey, Surrey Centre for Cyber Security, Guildford, UK \\ ${ }^{2}$ University of Luxembourg, Esch-sur-Alzette, Luxembourg \\ Email: s.wesemeyer@surrey.ac.uk, i.boureanu@surrey.ac.uk,zach.smith@uni.lu,h.treharne@surrey.ac.uk
}

\begin{abstract}
LoRaWAN (Low-power Wide-Area Networks) is the main specification for application-level IoT (Internet of Things). The current version, published in October 2017, is LoRaWAN 1.1, with its 1.0 precursor still being the main specification supported by commercial devices such as PyCom LoRa transceivers.

Prior (semi)-formal investigations into the security of the LoRaWAN protocols are scarce, especially for LoRaWAN 1.1. Moreover, amongst these few, the current encodings [4], [9] of LoRaWAN into verification tools unfortunately rely on much-simplified versions of the LoRaWAN protocols, undermining the relevance of the results in practice.
\end{abstract}

In this paper, we fill in some of these gaps. Whilst we briefly discuss the most recent cryptographic-orientated works [5] that looked at LoRaWAN 1.1, our true focus is on producing formal analyses of the security and correctness of LoRaWAN, mechanised inside automated tools. To this end, we use the state-of-the-art prover, Tamarin. Importantly, our Tamarin models are a faithful and precise rendering of the LoRaWAN specifications. For example, we model the bespoke nonce-generation mechanisms newly introduced in LoRaWAN 1.1, as well as the "classical" but shortdomain nonces in LoRaWAN 1.0 and make recommendations regarding these.

Whilst we include small parts on device-commissioning and application-level traffic, we primarily scrutinise the Join Procedure of LoRaWAN, and focus on version 1.1 of the specification, but also include an analysis of LoRaWAN 1.0. To this end, we consider three increasingly strong threat models, resting on a Dolev-Yao attacker acting modulo different requirements made on various channels (e.g., secure/insecure) and the level of trust placed on entities (e.g., honest/corruptible network servers). Importantly, one of these threat models is exactly in line with the LoRaWAN specification, yet it unfortunately still leads to attacks.

In response to the exhibited attacks, we propose a minimal patch of the LoRaWAN 1.1 Join Procedure, which is as backwards-compatible as possible with the current version. We analyse and prove this patch secure in the strongest threat model mentioned above.

This work has been responsibly disclosed to the LoRa Alliance, and we are liaising with the Security Working Group of the LoRa Alliance, in order to improve the clarity of the LoRaWAN 1.1 specifications in light of our findings, but also by using formal analysis as part of a feedback-loop of future and current specification writing.

\section{Introduction}

The Internet of Things is growing at a phenomenal rate, with billions of active devices. The implementation of connected sensors on small, embedded chips allows for data collection on an unprecedented scale.

The LoRa Alliance represents a collaboration between over 500 companies, producing a set of standards for IoT devices. The most notable of their contributions is the LoRaWAN specification for Long Range devices over a Wide Area Network (WAN). Over 80 million connected devices ${ }^{1}$ follow the LoRaWAN specification, meaning that its security is of key interest. In October 2017, the LoRaWAN specification was updated from version 1.02 to version 1.1 , containing significant updates to several sections. However, because many IoT devices run specialised hardware, the upgrade path has been very slow, with many devices remaining un-updated.

As such, both the LoRaWAN 1.0 and the LoRaWAN 1.1 specifications remain of interest to many communities. In this paper, we are interested in formally analysing their security and, particularly, the security of the LoRaWAN Join Procedure - in which a LoRaWAN Device uses a LoRaWAN Join Server to establish channel-keys used to communicate securely with a LoRaWAN Application Server. Although certain security concerns regarding the LoRaWAN Join have been raised in academic papers [1], in general, there has not been significant engagement of the LoRa Alliance with the academic community of formal security-analysis. This type of engagement is arguably desirable, considering for instance- the success of such collaborations in recent years, such as the formal modelling/analysis of TLS 1.3 [6] before its official release.

Related Work. There are several published discussions of vulnerabilities of LoRaWAN 1.0 Join [1], [9], using multiple different approaches. On the one hand, one of these [9] is semi-formal (using symbolic verification), yet it is arguably not a faithful analysis of LoRaWAN 1.0, as their modelling is over-simplified. On the other hand, [1] carries out a "pen-and-paper" analysis of the LoRaWAN 1.0 Join (i.e., not using formal methods), yet signals multiple security issues.

If the LoRaWAN 1.0 Join is not widely scrutinised, there are even fewer assessments of the security of LoRaWAN 1.1 Join [4], [5]. The frameworks used in these

1. https://lora-alliance.org/sites/default/files/2019-02/LORAWAN_ LIVE_MASTER_DECK_FINAL_FOR_RELEASE.pdf 
works do not always faithfully capture the additional details added in the latest version of the specification. Concretely, neither [4] nor [5] fully consider that the 1.1 Join is a four party authenticated key-exchange (AKE) protocol. In the case of [4], not only is it the case that the analysis is treated at the two-party AKE level and that Join Server is not modelled at all, but the choices of modelling abstractions remove important details and their specification files found online are incomplete. At the other end of the spectrum, Canard et al. [5] provide a formal, rigorous cryptographic model. However, they build on the 3ACCE model [2] (which is for proxied 3party AKEs), and fail to fully address the fourth party (i.e., the Application Server is not fully modelled).

Objectives. We aim to analyse the AKE protocols in the LoRaWAN 1.0 and 1.1 Join Procedures, as faithfully as possible, considering several threat models which accurately portray the real-world possibilities.

To do this, we make use of the Dolev-Yao [7] attacker: a man-in-the-middle who can intercept, redirect and modify any message, but is bound by the assumption of perfect cryptography. In other words, we operate in the space of symbolic verification/analysis of security protocols.

Contributions. The main contributions we present are as follows:

- We further the analysis of the LoRaWAN 1.0 Join, creating automated, tool-assisted proofs of the existence of attacks therein that had previously been be demonstrated only by hand in [1].

- We present faithful symbolic analyses, far beyond pre-existing efforts of this type [4], [9], of the LoRaWAN 1.1 Join, closely following its specification. We analyse the LoRaWAN 1.1 Join under multiple threat models, including the one declared by the LoRa Alliance. In these series of model, we also include the case where the Network Server (which acts as a proxy) may be partially dishonest.

To our knowledge, this is the first full symbolic analysis of the LoRaWAN 1.1 Join. Using this analysis, we demonstrate several security violations, including those in a model which closely follows that of the specification. Following from this, we discuss the shortcomings, inexactitudes and implications of the current specification for LoRaWAN 1.1 Join.

- Drawing on the above, we propose a new design of LoRaWAN 1.1 Join, which we call LoRaWAN 1.1 $L O R A \quad 3-A K A^{+}$. This is as backwards-compatible as possible with the current LoRaWAN 1.1 Join, and we prove using the Tamarin proving tool that LoRA $3-\mathrm{AKA}^{+}$is secure even in our strongest threat model.

Structure.The rest of the paper is structured as follows. In Section 2 we describe the Join procedure in the LoRaWAN 1.0 and 1.1 specifications, and give background on the Tamarin prover tool [15]). In Section 3 we introduce our threat models, as well as establishing our desired security goals. Section 4 contains a summary of the modelling and analysis of the two Join procedures. In Section 5 we present LoRA $3-\mathrm{AKA}^{+}$, our proposal for a new Join procedure which addresses the vulnerabilities identified. We then give the full results of our analysis in Section 6, which highlights the consequences of different modelling assumptions and threat models. In Section 7, we report of the discussions we had with the LoRa Alliance regarding to this work and its ramifications. Finally, we conclude in Section 8.

\section{Background}

In this section, we present the background and foundations to this work.

\subsection{The Join Procedure in LoRaWAN 1.1}

Protocol Description.The LoRaWAN 1.1 "Join procedure" is a four-party protocol [13], involving an end device $E D$, a Join Server JS, a Network Server $N S$ and an Application Server $A S$. The protocol relies primarily on the fact that $E D$ and $J S$ share two ${ }^{2}$ long-term symmetric keys: AppKey and NwkKey. The aim of the protocol is to use these long-term keys to establish a set of session keys for use in the record layer.

In Figure 1, we depict the Join Procedure in LoRaWAN 1.1, where -for simplicity- we omit certain lowlevel details, such as certain identifiers, message padding, and headers. Appendix B contains an enlarged figure with more details. The protocol proceeds as follows:

1) $E D$ sends to $J S$ a "Join Request" formed of a nonce DevNonce, relevant identifiers, along with a MAC of this information using the long-term key NwkKey.

2) This request is received by $N S$, who forwards it as-is (apart from network-level headers) to $J S$.

3) Upon successful verification of the MAC, JS produces a "Join Answer", which includes a "Join Accept", and a number of session keys. The crucial session keys are AppSKey and NwkSKey. We will discuss these keys' generation later.

- To create the "Join Accept", JS generates a new nonce JoinNonce, which it MACs and encrypts using the long-term keys shared with $E D$.

- In the "Join Answer", the AppSKey is encrypted with a long-term key that $J S$ shares with $A S$. - The "Join Answer" is sent by JS to $N S$.

4) The Network Server retains NwkSKey and forwards only the "Join Accept" part. From the "Join Accept", the device retrieves JoinNonce, and is then able to re-compute the session keys.

5-6) Later, in the first encrypted application-level message sent by $E D$, the application server receives either the encrypted AppSKey from the $N S$, or a sessionid with which to obtain the AppSKey from the $J S$. The $N S$ forwards this information along with the first application-level message. This will be further discussed later.

Keys Generated During the Join Procedure. During the Join Procedure, the Join Server (and later, the End Device) generates a set of session keys. The most significant of these are named AppSKey and NwkSKey ${ }^{3}$. These sessions keys are produced by encrypting with AES-128

2. There is a third, long-term shared key called JSIntKey.

3. There are two additional MAC keys for $N S$ and $E D$, namely, SNwkSIntKey and FNwkSIntKey. Our formal models capture all session keys. 


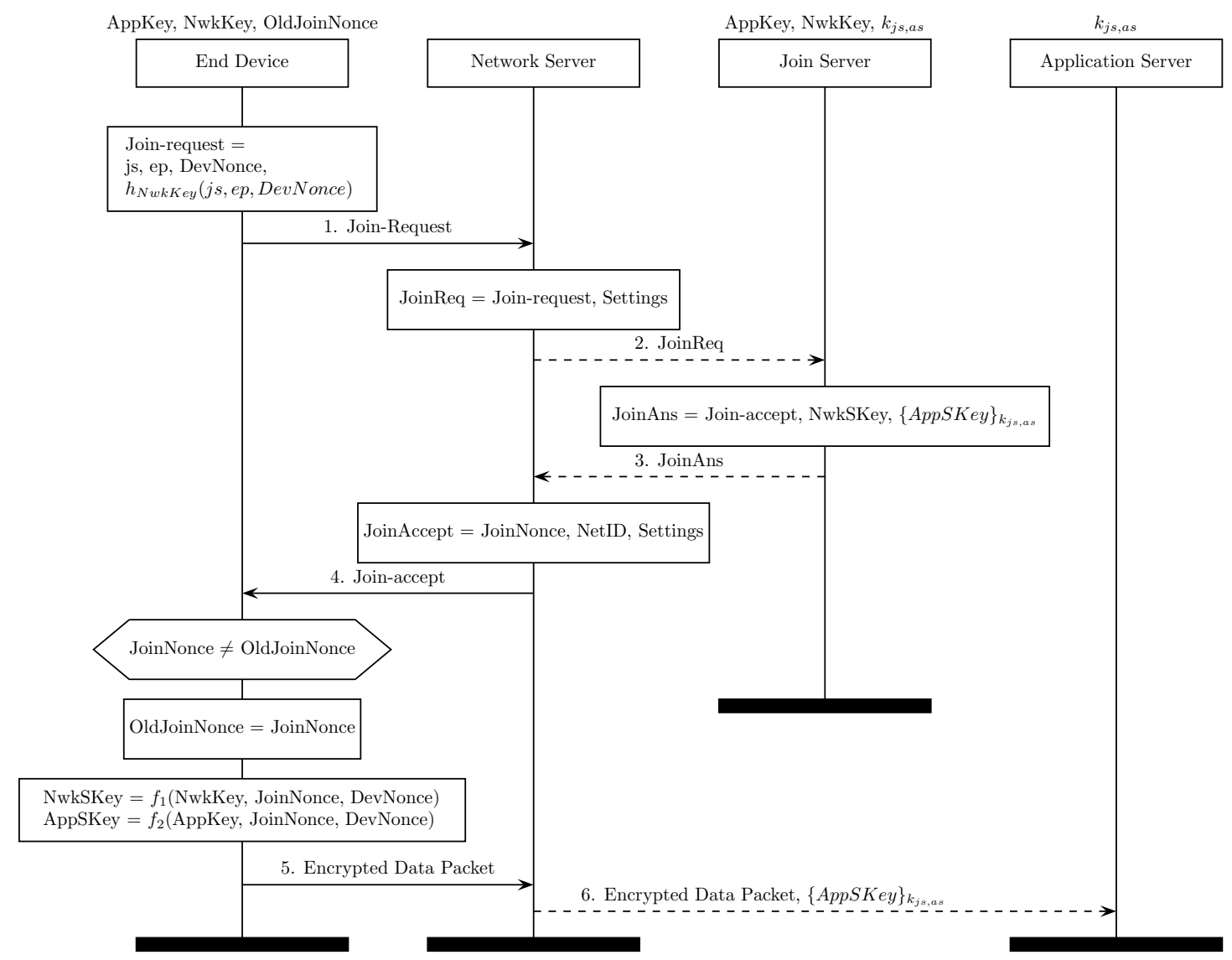

Figure 1: LoRaWAN 1.1 Join Procedure - Simplified View. Secure channels are displayed with dashed lines $--\rightarrow$

using the long-term keys AppKey and NwkKey, respectively. The following data is used in the key generation: DevNonce, JoinNonce and $J S$ 's identifier JoinEUI, along with specific message tagging ${ }^{4}$ (e.g., 0x02, 0x04, respectively). Each session key is used at the record layer for encrypting/decrypting application messages ${ }^{5}$ : NwkSKey is to be used by $N S$ and $E D$, and AppSKey by $A S$ and $E D$.

\subsection{LoRaWAN 1.1. Join Procedure Observations}

There are a few other aspects of the LoRaWAN 1.1 "Join Procedure" worth mentioning, which we detail below.

LoRaWAN 1.1 Channel Requirements. During the Join Procedure, as per the LoRaWAN 1.1 current specifications (i.e., 2017 version), the security of channels is assumed to be as follows: insecure channel between $E D$ and $N S$, insecure channel between $N S$ and $A S$, a secure channel between $N S$ and $J S$. Upon the end of this AKE, the intended channels are as follows: (a) confidential, authenticated and integral channel between $E D$ and $N S$ (obtained via encryption and MAC-ings); (b) insecure channel ${ }^{6}$ between $N S$ and $A S$, on which a

4. To not overload notation with different AES keys and different padding, on Fig. 1, we simply write NwkSKey is calculated using a function $f_{1}$ and AppSKey is calculated using a function $f_{2}$.

5. LoRa record-layer messages are not encrypted directly with the AppSKey, instead there is a bespoke KDF which uses AppSKey.

6. Concretely, line 1437 of the LoRaWAN 1.1 specifications mentions that,a-priori, there is no secure channel between $N S$ and $A S$. correct behaviour is one where $N S$ is proxying AppSKeyencrypted messages between $E D$ and $A S$. There is also a secure channel between $A S$ and $J S$, which can optionally be used to deliver the AppSKey.

Note the insecure channels between $N S$ and $A S$. More precisely, the main LoRaWAN 1.1 specifications and the backend specifications as they stand (in 2017), make no explicit, clear or mandatory requirement of integrity or of confidentiality with respect to the channel between $N S$ and $A S$ during the Join or thereafter, at the application level.

Bespoke Nonce-generation in LoRaWAN 1.1.In LoRaWAN 1.1, DevNonce is not sampled uniformly at random over its domain. Instead it is produced as a strictlyincreasing counter. $N S$ keeps a tally of DevNonces per device, and checks that it is increasing (see page 52 of the specifications). Similarly, the JoinNonce (which is 24 bits long) is produced by the increasing a counter, kept up-to-date for each device.

Alternative AppSKey-deliveries in LoRaWAN 1.1.There are in fact two ways in which AppSKey can be delivered to $A S$ : (1) by the $N S$ who would forward it with the first application message (which is shown on Fig 1); (2) by the $J S$, upon request. For the latter, the $J S$ needs to have generated a SessionKeyID and that be sent alongside the encrypted AppSKey in the Join Answer message, to the NS. If a SessionKeyID is given, the $N S$ can forward this alongside the first data packet. In order to retrieve the encryption key, $A S$ contacts $J S$, who delivers the corresponding AppSKey. 
Figure 11, in the appendices, shows this second means of AppSKey delivery.

The specification does not indicate which approach is preferred. In this second case, $A S$ cannot immediately decrypt messages sent from $E D$.

In both cases, it is not clear from the specifications whether $A S$ is to check if the first data-packet/applicationlevel message does indeed decrypt correctly with the delivered AppSKey and how the protocol continues in case of failure.

\subsection{The Join Procedure in LoRaWAN 1.0}

Now, we explain the LoRaWAN 1.0 Join procedure protocol. We use as a reference the LoRaWAN 1.0.2 version of the Join Procedure [14]). We focus on highlighting the differences with the LoRaWAN 1.1 Join relevant to the work herein.

\subsection{LoRaWAN 1.0 Join vs. LoRaWAN 1.1 Join}

Compared to the LoRaWAN 1.1 Join, the LoRaWAN 1.0 Join considers only three active roles, End Devices (ED), a Network Server $(N S)$, and the backend Application Server $(A S)$ : i.e., there is no Join Server. The role played by the Join Server in the LoRaWAN 1.1 Join is encapsulated in the LoRaWAN 1.0 Join into the Network Server logic. Also, there are fewer shared long-term keys used in the AKE protocol. In the description below, we denote the singular long-term shared key as AppKey.

Much like in the case of 1.1, the Application Server is not active during the Join protocol, instead receiving the derived AppSKey from the Network Server.

Unlike in the LoRaWAN 1.1 Join, in the 1.0 Join the DevNonce and JoinNonce are sampled uniformly at random over their domain. Yet, DevNonce is only 16bit long, leading to a birthday paradox-style attack for LoRaWAN 1.0 (i.e., high chance of repetitions over sessions) [1].

\subsection{The Join Procedure in LoRaWAN 1.0}

Figure 2 shows the messages exchanged in the LoRaWAN 1.0 Join.

The protocol proceeds as follows:

1) The End Device sends a "Join Request" message, consisting of a nonce DevNonce along with identifiers for $E D$ and $A S$. The message is MAC-ed using the long term key AppKey.

2) After checking the freshness of DevNonce $E D^{7}, N S$ replies with a "Join Accept" message. This message contains identifiers for $N S$, a fresh nonce AppNonce, and a collection of parameters to be used for further communication. This message is encrypted using $\mathrm{AppKey}^{8}$, and a MAC of the plaintext is sent back alongside this encryption.

3) $E D$ and $N S$ can now calculate the session keys NwkSKey and AppSKey, as AES-128 encryptions under AppKey of AppNonce and DevNonce together with specific tagging (e.g., 0x01, 0x02). To make the figure more readable, we write $f_{1}$ and $f_{2}$ respectively instead of aes(AppKey; $\left.0 x 01 \| \ldots\right)$, aes(AppKey; 0x02||...).

\subsection{The Tamarin Prover}

The Tamarin prover [12] is a popular verification tool that supports symbolic analysis [3] of security protocols in the Dolev-Yao model [7] in the unbounded setting.

Tamarin models are transition systems over a multisorted term algebra, operating on the semantics of multiset rewriting logic [8]. Security properties to be analysed are expressed in a guarded first-order logic quantifying over atomic facts declared in the model. For the analysis that we are interested in (i.e., that of trace properties), Tamarin performs a backwards reachability analysis: beginning

7. "For each end-device, the network server keeps track of a certain number of DevNonce values used by the end-device in the past, and ignores join requests with any of these DevNonce values from that enddevice." [14]

8. Technically, the message is decrypted using this key, so that the end device needs only implement the primitive for encryption

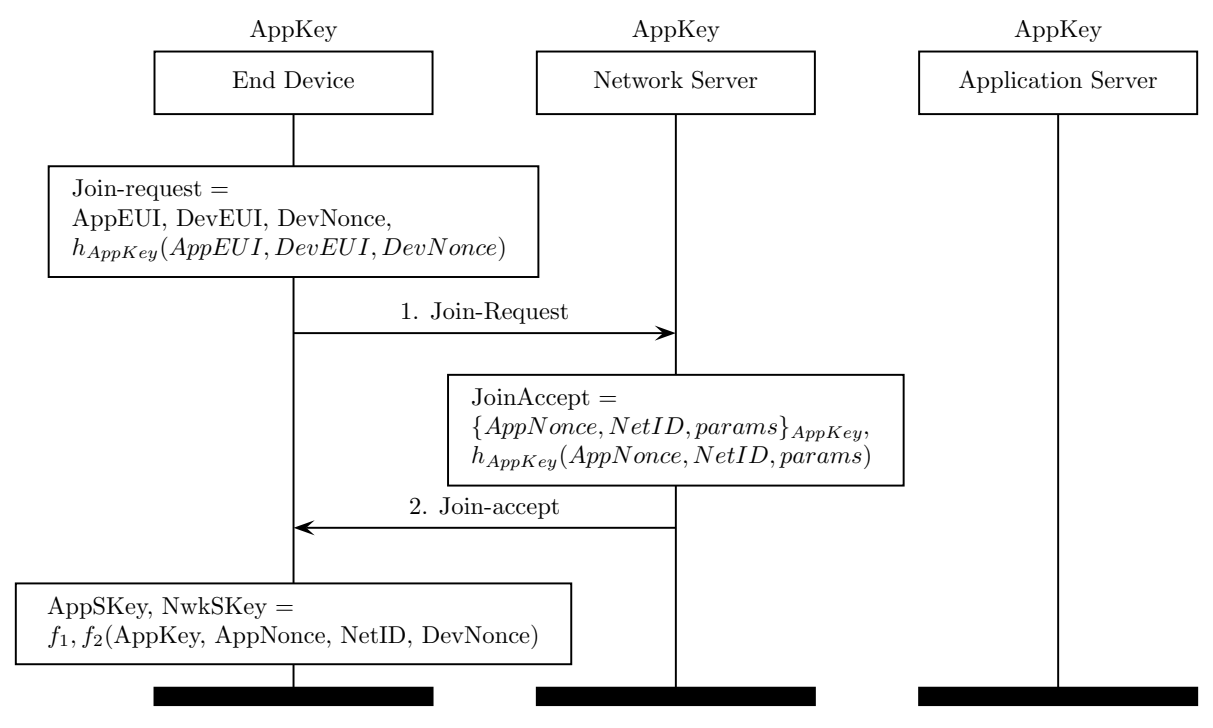

Figure 2: LoRaWAN 1.02 Join Procedure 
from a state representing a counterexample to a security claim, it then attempts to build a trace in backwards steps to the empty state. The existence of such a trace represents a violation of the desired security goal. Generally, Tamarin is used as an interactive tool, where the user guides the proof search.

Tamarin Oracles. Tamarin supports various heuristics to cover the search-space yielded by the constraint-solving problem underlying the analysis. These heuristics, similarly to user interaction, determine which rules should be prioritised during the search. To this end, the user can rank different rules: higher ranked rules are triggered with priority. Such a set of rule rankings yields a bespoke search heuristic, named an "oracle", which can be passed to Tamarin [15]. These oracles only influence termination and proof-search, not the result, such that soundness is preserved.

\section{Threat Models \& Security Properties}

\subsection{Threat Models}

Balance of Trust \& Security in the LoRaWAN 1.1 Specifications. We consider three threat models, which we name $\mathcal{M}_{A S-N S \text {-Secure }}, \mathcal{M}_{\text {LoRa1.1SpeC }}$,

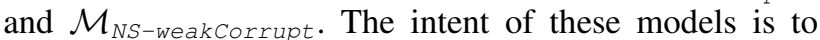
balance, as well as probe, the trust assumptions and the security requirements made by the LoRaWAN specifications. Concretely, we differentiate these models based on the amount of trust placed in the Network Server, as well as the security of channels between $N S$ and the backend.

\begin{tabular}{|c|c|c|c|}
\hline & \multicolumn{2}{|c|}{ Channel Security } \\
\hline & & High & Low \\
\hline$N S$ & High & $\mathcal{M}_{\text {AS-NS-Secure }}$ & $\mathcal{M}_{\text {LoRa } 1.1 \mathrm{Spec}}$ \\
\hline trust & Low & - & $\mathcal{M}_{\text {NS-weakCorrupt }}$ \\
\hline
\end{tabular}

$\mathcal{M}_{\text {LoRa1.1Spec }}$ is the threat model exactly implied by the LoRaWAN 1.1 specifications for the Join Procedure. In this case, there is a high level of trust on $N S$. More specifically, as per the specifications, we consider all parties be honest. As per the specs, we model the the channels between $N S$ and $J S$ and between $A S$ and $J S$ as secure, but the channel between $N S$ and $A S$ as insecure, i.e., as the specifications do not require it be secure.

$\mathcal{M}_{\text {AS-NS-Secure }}$ has the same level of trust as $\mathcal{M}_{\text {LoRa1.1Spec }}$, i.e., the $N S$ and all other parties are considered honest. However, for $\mathcal{M}_{\mathrm{AS}-\mathrm{NS} \text {-Secure }}$, we consider the channel between $N S$ and $A S$ to be secure. As a result, $\mathcal{M}_{\mathrm{AS}-\mathrm{NS} \text {-Secure }}$ is a weaker threat model than $\mathcal{M}_{\text {LoRa1.1Spec }}$. Security analysis in this model is arguably adequate, as a proprietary $A S$ might be commissioned with specific security measures (i.e., authentication, confidentiality and integrity) in its communication with the $N S$.

$\mathcal{M}_{\text {NS-weakCorrupt }}$ is a stronger threat model than $\mathcal{M}_{\text {LoRa1.1Spec }}$. Concretely, $\mathcal{M}_{\text {NS-weakCorrupt }}$ is in fact the same as the $\mathcal{M}_{\text {LoRa1.1Spec }}$ model except that the channel between $N S$ and $J S$ has become insecure. In particular, in $\mathcal{M}_{\text {NS-weakCorrupt }}$, the $N S$ behaves as per its specification, and the attacker has extra powers only in that it has compromised the security of the channel between the $N S$ and the JS. Arguably, this is a weak form of compromising the $N S$. That is, the model $\mathcal{M}_{\mathrm{NS} \text {-weakCorrupt }}$ does not take away this entire trust assumption in the LoRaWAN 1.1 Join (i.e., $N S$ does continue to behave follow the protocol); instead, $\mathcal{M}_{\mathrm{NS} \text {-weakCorrupt }}$ probes the implications of even the smallest compromise of this assumption.

In our formal modelling (in Tamarin), insecure channels are implicitly public and thus accessible to the DolevYao attacker for active manipulation.

So, the $\mathcal{M}_{\text {LoRa1.1Spec }}$ model is the baseline (in line with the trust and security of the LoRaWAN specifications), $\mathcal{M}_{\mathrm{NS} \text {-weakCorrupt }}$ is a weakening of trust assumptions in $\mathcal{M}_{\mathrm{LOR} \text { (1.1Spec }}$, whereas $\mathcal{M}_{\mathrm{AS}-\mathrm{NS} \text {-Secure }}$ is a strengthening of the secure-setup requirements in $\mathcal{M}_{\text {LoRa1.1Spec }}$.

Threat Models for LoRaWAN 1.0. Note that whilst all the above models apply to the LoRaWAN 1.1 Join, they do not apply to the LoRaWAN 1.0 Join. Concretely, $\mathcal{M}_{\text {NS-weakCorrupt }}$ rests on the security of the channel between $N S$ and $J S$, but this channel is not present in the LoRaWAN 1.0 Join (as $J S$ does not exist therein); and in the 1.0 Join, the $A S$ is completely inactive, so $\mathcal{M}_{\text {LoRa1.1Spec }}$ and $\mathcal{M}_{\text {AS-NS-Secure }}$ do not apply.

\subsection{Properties Analysed}

For our verification, we encode various agreement and synchronisation properties [11]. Such goals are commonplace for AKE protocols: e.g., weak agreement, noninjective agreement, injective agreement, synchronisation and secrecy of the established key (key secrecy, for short). The main difference is that, for LoRaWAN 1.1, these are extended to encompass not the standard two parties, but rather three or four parties, as demanded by the LoRaWAN Join procedures.

As we give details of our models, which themselves make the properties more specific, we further explain the precise nature of certain formulations of our properties.

\section{Security Analysis of the LoRaWAN Joins}

In this section, we apply our threat models and examine the security of the LoRaWAN 1.0 and 1.1 Join Procedures. We give an overview of our Tamarin models and summarise the most important observations and their consequences.

All of our Tamarin models can be found via the website https://loraverif.github.io/.

\subsection{Modelling of LoRaWAN 1.0 Join}

We first go over our analysis of the LoRaWAN 1.0 Join procedure. Note that for this protocol we consider only the $\mathcal{M}_{\text {LoRa1.1Spec }}$ threat model. This is because there is no Join Server, changing the assumptions on secure channels.

We also do not model confusion freeness, as in this case it is subsumed entirely by the synchronisation goal, sync_ED_NS, defined in the following subsection.

Comprehensively \& Closely Modelling the LoRaWAN 1.0 Specs in Tamarin. Figure 2, presented earlier, shows a simplified version of the LoRaWAN 1.0 join procedure. However, our Tamarin model follows the 
specification much more closely. Appendix A contains an example of the level of detail captured - which includes padding and optional parameters.

Nonce Freshness \& Short Nonces. The specification states that $N S$ should keep a tally of nonces sent by $E D$, performing necessary freshness checks. Our model fully captures this.

We also simulate the fact that nonces are only over generated small domains (DevNonce $\in\{0,1\}^{16}$ and JoinNonce $\in\{0,1\}^{24}$ ). As detailed in the next paragraph, we devise a manner of producing nonces in a cyclic manner. This clearly under-approximates true nonce generation (which would be done by simply using a "Fresh" sort), however it encapsulates the fact that collisions of nonces are likely. It is arguably unusual for symbolic methods to encode this fact, however LoRaWAN 1.0 insecurities stemming from these collisions are known [1]. So, our model aims to see if we can find attacks based on this same short-nonces shortcoming.

Our modelling approach in this case is to encode the set of possible values as constant terms (i.e. ' 1 ', ' 2 ', ...) rather than abstract fresh terms. This set of values is encoded into the specification of $E D$. When a value is to be chosen, we take advantage of Tamarin's multiset builtin, which permits nondeterministic selection of an element from a multiset. To verify the soundness of this "small-domain" encoding, we prove two lemmas:

(a) two_join_requests_distinct_nonces_device, stating a device can always send two (or more) Join Requests with different nonces. This ensures that our encoding does not restrict the behaviour too coarsely;

(b) two_join_requests_same_nonce_device, stating that a device can send two Join Requests with the same nonce, demonstrating that our modelling can indeed capture the shortcomings of short-nonce repetition.

\subsection{Analysis of LoRaWAN 1.0 Join}

We first present the main security properties we encode and verify.

Key-Agreement Properties. Firstly, we focus on standard agreement properties for AKE protocols, as seminally introduced by Lowe [10]. To this end, we encode the following properties with corresponding intuitions:

(1) weak agreement (wa_ED_NS) - whenever a device $E D$ has completed the Join Procedure with a network server $N S$, then some execution of $N S$ has "recorded" to have run the Join with said $E D$;

(2) non-injective agreement (nia_ED_NS) - whenever a device $E D$ has completed the Join Procedure with a Network Server $N S$, and ED's transcript contains certain messages, then some execution of $N S$ has "recorded" to have run the Join with said ED with the same said messages;

(3) injective agreement (ia_ED_NS) - whenever a device $E D$ has completed an execution $d$ of the Join Procedure with a network server $N S$ and $E D$ 's transcript for execution $d$ contains certain messages, then there is a unique execution $n$ of $N S$, which records a Join Procedure with said $E D$, and the same said messages that appear in $E D$ 's execution.
(4) synchronisation (sync_ED_NS) - in any full Join execution there exists an $E D$ and a $N S$ who both have matching views of the transcript of the execution.

(5) session-key secrecy (key_secrecy) - if a secret key $x$ is established at timepoint $\mathrm{i}$, then either the adversary does not know $x$, or the agent who established said key has been compromised beforehand.

Freshness Failure. The main specification encoded for checking freshness and identifying potential replay attacks is the following fifth property:

(6) replay-attack existence (replay) - there can exist two Join executions, $i$ and $j$, in which DevNonce De $_{i}=$ DevNonce $_{j}$ and AppNonce $_{i}=$ AppNonce $_{j}$ (i.e., in which both ED's nonce and $N S$ ' nonce repeat themselves).

Analysis Results for LoRaWAN 1.0. We now present some details and the results of our analysis for the properties above, performed using the Tamarin prover tool. To carry out the verification, we created an oracle to improve the automatic navigation of the search-space.

The results show that 4 out of the 6 properties examined for agreement and synchronisation in LoRaWAN 1.0 Join are violated. We provide a summary in the table below.

\begin{tabular}{|l|l|}
\hline Property & Result \\
\hline \hline (1) weak agreement (wa_ED_NS) & holds \\
\hline (2) non-injective agreement (nia_ED_NS) & falsified \\
\hline (3) injective agreement (ia_ED_NS) & falsified \\
\hline (4) synchronisation (sync_ED_NS) & falsified \\
\hline (5) key secrecy (key_secrecy) & holds \\
\hline (6) replay-attack resistance (replay) & falsified \\
\hline
\end{tabular}

Table 1: Verification Results of the LoRaWAN 1.0 Join

We now go over some of the results contained in Table 1. Further execution details are given in Section 6.

Interpretation of Attacks. We note that the second and fourth failings, of non-injective agreement and synchronisation respectively, come down to equivalent traces. This trace shows that the main crux of the failure is that DevNonce is not used in the LoRaWAN 1.0 Join Response. We note that this behaviour is rectified in the LoRaWAN 1.1 Join.

The fact that replay-attack resistance fails indicates that even with checks by the $N S$ on the freshness of DevNonce, the protocol does fail to catch replay attacks. This is a result of the small size of the set of possible nonces. Namely, the trace shows that it is possible for there to be a session $i$ in which the attacker can replay an old DevNonce from a session $j$ and, in session $i$, the $N S$, AppNonce also coincides with the AppNonce in session $j$. As a result, the AppSKey established in each of two traces is the same. Given how the encryption of the recordlayer messages works in LoRaWAN 1.0, this means that the attacker can retrieve elements of the plaintext of the record-layer messages from the two sessions $i$ and $j$. Note that the replay attack we exhibit by this was shown by hand in 2018 [1]; however, this is the first time this replay was automatically found by a protocol-verification tool.

As well as verifying the soundness of our "smalldomain" nonce approach, we also verified that this mod- 
elling is complete - that the attacker cannot always perform this replay attack. To this end, we prove two additional lemmas, which demonstrate that there are also traces in which only one nonce (be it the DevNonce or the AppNonce) repeats itself.

\subsection{Modelling of LoRaWAN 1.1 Join}

Comprehensively \& Closely Modelling the LoRaWAN 1.1 Specifications in Tamarin. We follow the specifications of the LoRaWAN 1.1 Join closely, encoding in Tamarin levels of details far beyond the simplified description given in Figure 1. For instance, the Tamarin code-snippet shown in Figure 3 shows part of the specification of the JoinServer, which contains details about sessions keys, padding, and counters.

In order to justify the choices made in our modelling, our Tamarin files contain references to the line/page of the LoRaWAN specifications which justify our choice of encoding.

Modelling the Join \& Data Packets in the LoRaWAN 1.1. The LoRaWAN 1.1 Join Procedure finishes before the first data packet is sent (i.e., the keys are established before this packet is sent). However, as explained in Subsection 3.2, we wish to verify key-agreement properties with respect to all four parties involved, but $A S$ receives the AppSKey only when first data packet is delivered (or shortly afterwards, depending on the delivery method). To this end, we model the full Join Procedure, plus the first data packet.

Variations of the LoRaWAN 1.1 Specifications Modelled. The specification contains no clear requirement on the $A S$ being able to decrypt the first data-packet sent to it. To this end, we model two variations of the LoRaWAN 1.1 Join:
- The "Desync-Model", which encodes the LoRaWAN 1.1 specification precisely (where the $A S$ will not end in "error" if it receives the first application message malformed);

- The "Sync-Model", which encodes a slight tightening of the LoRaWAN 1.1 specifications, whereby the $A S$ will end in "error" if it receives a malformed first application message.

For each such "sync"/“desync" setting, we encode the two cases discussed in Subsection 2.2 as to how $A S$ receives the AppSKey. This yields:

- "AppSKey-from-NS", which encodes that the NS delivers the (JS-encrypted) AppSKey directly to the $A S$, with the first application-level message (as per Figure 1);

- “AppSKey-from-JS”, which encodes that the JS delivers the AppSKey to the $A S$, upon the latter's request yielded after the first application-level message arrived at the $A S$ (as per Figure 11).

Encoding Different Threat Models. For both the Desync-Model and the Sync-Model, as well as for both the Appskey-from-NS and Appskey-from-JS variations, we create four different Tamarin files, each encapsulating one of the three threat models $\mathcal{M}_{\text {LoRa1.1Spec }}$, $\mathcal{M}_{\text {NS-weakCorrupt }}$ and $\mathcal{M}_{\mathrm{AS}-\mathrm{NS} \text {-Secure }}$, presented in Subsection 3.1.

Consequently, to capture all these different scenarios, we include a total of 12 Tamarin files for LoRaWAN 1.1. Note that this is mainly done for convenience, with the vast majority of the Tamarin code shared between models, and only minor changes in key locations. This separation allows us to pinpoint which "configurations" of LoRaWAN 1.1 lead to attacks.

Restrictions. In order to improve the tractability of our analysis in the most severe threat model,

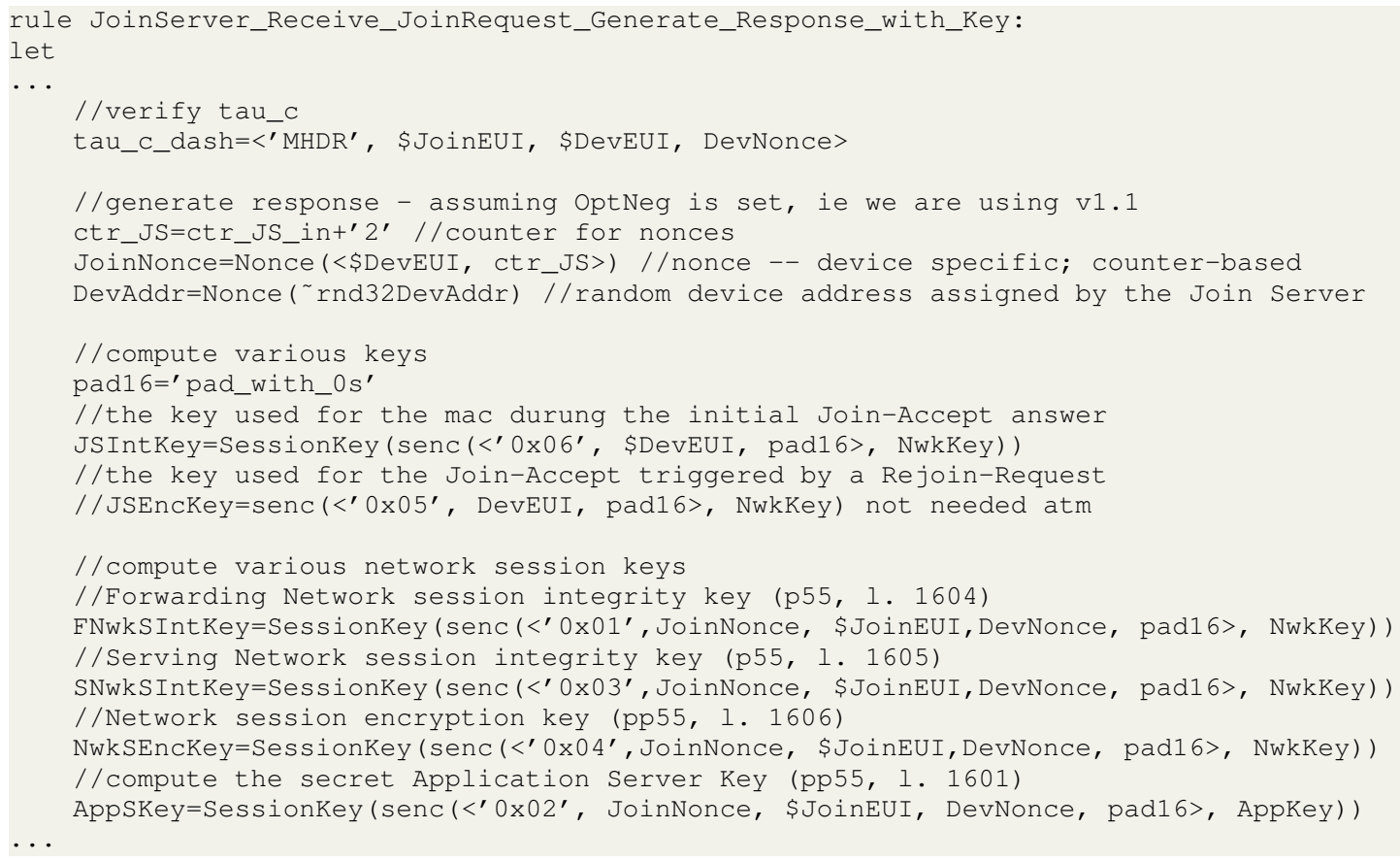

Figure 3: Code Snippet of Tamarin rule for LoRaWAN 1.1 Join Procedure 
$\mathcal{M}_{\text {NS-weakCorrupt }}$, we include a series of restrictions on the maximum number of Join Requests and the maximum number of Join Responses. This means that, in this strongest model and for this property, we look to see if there is any attack within a maximum number of spanned sessions for one given Device and one given Join Server. However, we do not bound the attacker's powers in other ways, such as number of nonces or messages. Concretely, for the LoRaWAN 1.1 Join in the $\mathcal{M}_{\text {NS-weakCorrupt }}$ model, proofs were carried out with a maximum number of Join Responses and Requests set to 4 and 3 respectively. These values were chosen with the tradeoff between computational cost and faithfulness of the model in mind. However, for exhibiting an attack, we found counterexamples of the properties in $\mathcal{M}_{\text {NS-weakCorrupt }}$ even with this set to 2 .

Weakly-typed Models vs. Source Lemmas. Specifically for the case of proving properties on the $\mathcal{M}_{\text {NS-weakCorrupt }}$ models, we wrote several Tamarin source lemmas (see [15]). However, these proved ineffective. Instead, we achieved better performance by weaklytyping ${ }^{9}$ the models. Nonetheless, we did this in a parsimonious way, i.e., we only declared: (a) a specific sort Nonce as a subsort of Fresh, to exclude typing-attacks on nonces; (b) a specific format for data-packages after the Join finished, to stop injection of *any* possible term therein. Arguably, these two weak-typings are realistic and cannot exclude mainstream key-agreement attacks. These together with the aforementioned restrictions allowed us to disprove (different versions) of confusion freeness in the strongest attacker model $\mathcal{M}_{\mathrm{NS} \text {-weakCorrupt }}$.

Counter-based Nonces. As described in Subsection 2.2, the LoRaWAN 1.1 specifications replace nonces with counter-based bitstrings both on the $E D$ and on the $J S$, which the latter having device-specific counters. We model this in Tamarin, using the multiset builtin. Counters are modelled as multisets over a domain of a single element (i.e. the counter can have values ' 1 ', ' 1 ' $+{ }^{\prime} 1$ ' $\ldots$ ), incrementing on each use. Both $E D$ and $J S$ record the last accepted value of their partner's counters, and will only accept a message if the counter is increasing.

\subsection{Security Goals of LoRaWAN 1.1 Join}

We now present the main security properties we encode and verify.

Firstly, to encode equivalent properties as in the LoRaWAN 1.0 Join, we look at agreement properties between $E D$ and $J S$. To this end, recall that the role of $N S$ in the LoRaWAN 1.0 Join is assumed in LoRaWAN 1.1 by $J S$. So, with the equivalent meaning as those presented in Subsection 4.2, we encode:

(1) key secrecy for the NwkSKey and AppSKey (key_secrecy)

(2) weak agreement between ED and $J S$ (wa_ED_JS)

(3) non-injective agreement between ED and JS (nia_ED_JS)

(4) injective agreement between ED and JS (ia_ED_JS)

9. Such techniques have been used before in Tamarin-verification when large systems (such as TLS1.3) were encoded and analysed [6].
Secondly, we move to encoding AKE requirements linked specifically to the LoRaWAN 1.1 Join, i.e., between the Devices and the Application Server. We specify the following lemmas:

(4) weak agreement between $E D$ and $A S$ (wa_ED_AS) which encodes that whenever a device $E \bar{D}$ has completed the LoRaWAN 1.1 Join Procedure allegedly with $A S$ then some execution of $A S$ has "recorded" running with $E D$.

We note, once again, that (in our models and in the LoRaWAN 1.1 specification), an $A S$ "ascertains" that it is communicating with a specific $E D$ only when said $A S$ receives the first (encrypted) data packet and the corresponding AppSKey to decrypt it, as -beforehandthe $A S$ is not alive during the actual key-establishment run by the $E D$ and $J S$ (via $N S$ ). Recall that, to capture error-handling if the packet-decoding by the $A S$ fails, we encode the two variations "Desync-Model" and "Sync-Model" of the LoRaWAN specifications. In both of these models, we look at the synchronisation sync_ED_AS property below:

(5) synchronisation between ED and $A S$ (sync_ED_AS) - which checks if there is an execution in which $E D$ sends a data-packet, yet the data-packet received by $A S$ cannot be decoded.

Moreover, we also encode agreement properties similar to sync_ED_AS but slightly stronger. Namely, we check 2-party and 3-party agreement properties with respect to data packets and/or AppSKeys, to check not only if the $A S$ is (un)able to decode a data-packet (as per sync_ED_AS), but also if an $A S$ could potentially be adversarially confused as to which data-packet and/or AppSKey is associated with which ED. We call these agreement properties confusion freeness and describe several of their flavours below.

(6) two-party confusion-freeness w.r.t. packets (CF_ED_AS_packet) - which encodes that if a device $\bar{E} D$ finishes a session $i$ by sending an encrypted data-packet $p_{i}$ to an application server $A S$, then if the application server $A S$ gets this data-packet $p_{i}$ it believes it originates from this device $E D$.

(7) two-party confusion-freeness w.r.t. AppSKey (CF_ED_AS_packet_key) - which encodes the same as CF_ED_AS_packet w.r.t. the AppSKey: i.e., if a device $E D$ finishes a session $i$ with a given AppSKey ${ }_{i}$, then if the application server $A S$ receives $\mathrm{AppSKey}_{i}$, it correctly believes it is meant for communicating with $E D$.

(8) three-party confusion-freeness w.r.t. AppSKey (CF_ED_JS_AS_key) - which encodes the same as CF_ED_AS_packet_key, factoring in the $J S$ as well: i.e., the view on a given AppSKey is synchronised correctly between a given $E D \mathrm{~s}$, the $J S$ and the $A S$.

As with LoRaWAN 1.0, we analyse additional lemmas, primarily to check the correctness of our modelling, or for auxiliary security properties (e.g., that the keysetup/commissioning we model between Devices and the Join Server, is secure). However, as they are not pertinent to the analysis, we omit details on those here.

Last but not least, we recall that we run our verification for these properties on both the "Desync-Model" 


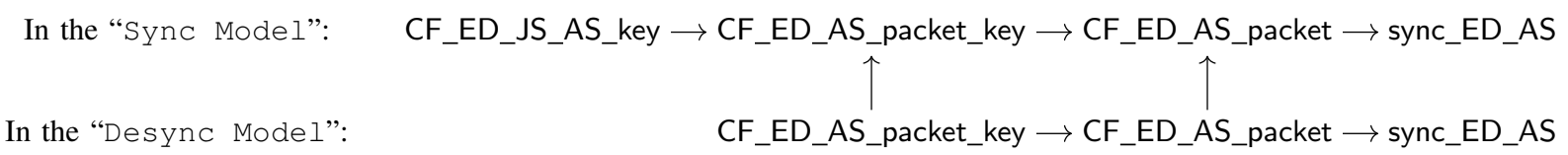

Figure 4: Hierarchy of Confusion-Freeness and Synchronization Goals

and "Sync-Model" of the specifications, in each of the three threat models $\mathcal{M}_{\text {LoRa1.1Spec }}, \mathcal{M}_{\mathrm{NS} \text {-weakCorrupt }}$ $\mathcal{M}_{\text {AS-NS-Secure, }}$, with two different methods for distributing AppSKey. To this end, we created various bespoke oracles for the respective analyses.

\subsection{Hierarchy of Properties Analysed}

The relationships between our various security properties are as follows:

Agreement Properties. Our agreement properties are directly drawn from the definitions of Lowe [10]. Although the protocols we analyse are not two-party, the agreement results work on a pairwise basis. Hence we have that injective agreement is stronger than non-injective agreement, which in turn is stronger than weak agreement.

Confusion-Freeness Properties. The simplest of the confusion-freeness properties is CF_ED_AS_packet. CF_ED_AS_packet is stronger than sync_ED_AS, as it looks not just at the $A S$ decoding the packets, but also the $A S$ attributing the device address inside said packets to a device. This process is tied in with the format of the application-layer messages per se and (also) with how the device address (that appears in these messages) is fed ${ }^{10}$ to the $A S$. In other words, analysing CF_ED_AS_packet also looks at potential confusion of device identities.

Also, in this vein, CF_ED_AS_packet is weaker than CF_ED_AS_packet_key, as the latter requires agreement on AppSKey as well as the packets sent to be associated with that AppSKey. In fact, note that the confusion-freeness properties (CF_ED_AS_packet_key, CF_ED_JS_AS_key, ) that require agreement on both the AppSKeyas well as the data-packet, logically, are not truly needed in the "Desync-Model": if the $A S$ is able to report failure when decoding the packet, it is less relevant if it receives an incorrect AppSKey. As such, we check the CF_ED_AS_packet_key, CF_ED_JS_AS_keyonly in the "Sync-Model".

Some of the confusion-freeness properties require three parties to agree, compared to the simpler case of just two parties. Clearly, the former are stronger requirements than the latter. Not only that, but given the modelling, if the 3 parties at hand agree on an AppSKey (i.e., CF_ED_JS_AS_key holds), then necessarily the $A S$ and $E D$ would agree on the packet as well (i.e., CF_ED_AS_packet_key holds); this is because the first agreement over 3 parties also binding over identification of the device (ED's EUI and ED's address), in a way that links in with the packets sent and received by the EDand the $A S$, respectively.

Other Relationships. Although not necessarily true in the universal setting, an attack on key secrecy for the LoRaWAN Join protocol immediately results in a

10. For details, see e.g., pages 12, 18, 19 of the backend specifications. scenario where the adversary can forge data packets (and the associated MACs). Such an attack would thus entail violations of the majority of the other properties checked.

We note that the "Desync Model" encodes a weaker system than the "Sync Model". So, for the formulae that make sense to be checked in both (e.g., CF_ED_AS_packet and sync_ED_AS), we have an implication from "Desync"-based formulations to "Sync "-based formulations.

Unlike in the case of "Desync" vs. "Sync", hierarchical lines cannot be drawn in between properties holding in the variations of the models w.r.t. AppSKey deliveries: that is, the "AppSKey-from-NS" and "AppSKey-from-JS" are incomparable with respect to these properties holding:

prop. in "AppSKey-from-NS" $\stackrel{\leftrightarrow}{\leftarrow}$ prop. in "AppSKey-from-JS"

Finally, if any property holds in the $\mathcal{M}_{\mathrm{NS} \text {-weakCorrupt }}$ model, then it will hold in the $\mathcal{M}_{\text {LoRa1.1Spec }}$ model. If any property holds in the $\mathcal{M}_{\text {LoRa1.1Spec }}$ model, then it will hold in the $\mathcal{M}_{\mathrm{AS}-\mathrm{NS}-\text { Secure }}$ model:

$\mathcal{M}_{\text {NS-weakCorrupt }} \Rightarrow \mathcal{M}_{\text {LoRa1.1Spec }} \Rightarrow \mathcal{M}_{\text {AS-NS-Secure }}$

In Figure 4 we present the part of our hierarchy of properties that does not trivially follow from Lowe's agreement lattice, but is rather dictated by our threat and system models.

\subsection{Analysis Results}

We verified the above properties in all our threat models introduced in Subsection 3.1: $\mathcal{M}_{\mathrm{AS}-\mathrm{NS}-\text { Secure }}$, $\mathcal{M}_{\text {LoRa1.1Spec }}$, and $\mathcal{M}_{\text {NS-weakCorrupt }}$.

We first report on the analysis of $E D-J S$ properties. Given the presence of a secure channel between the $N S$

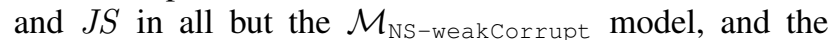
implicit assumption that $E D$ is honest in these security claims, these properties hold in the $\mathcal{M}_{\text {LoRa1.1Spec }}$ and $\mathcal{M}_{\text {AS-NS-Secure }}$ cases. However, these properties do not hold in the $\mathcal{M}_{\mathrm{NS} \text {-weakCorrupt }}$ threat model, when the adversary is able to manipulate the (mostly plaintext) messages on this channel.

We now present our verification results for the properties between the $E D$ and the $A S$. Table 2 contains a simplified view of our results, while Table 3 in Section 6 contains the outcomes of all of the analysis performed. Due to the number of different modelling scenarios, we focus our discussion on a few key cases.

We begin with the scenario which most closely follows the LoRaWAN specification. This is the $\mathcal{M}_{\text {LoRa1.1Spec }}$ threat model, using the Desync-Model encoding. This model is weaker than the Sync-Model, as the $A S$ accepting potentially malformed messages enables some attacks. 


\begin{tabular}{|l|l|l|l|}
\hline & sync_ED_AS & CF_ED_AS_packet_key & CF_ED_JS_AS_key \\
\hline \hline $\mathcal{M}_{\text {LoRa1.1Spec }}$ & fails & fails & fails \\
\hline $\mathcal{M}_{\text {NS-weakCorrupt }}$ & fails & fails & fails \\
\hline $\mathcal{M}_{\text {AS-NS-Secure }}$ & holds & holds & holds \\
\hline
\end{tabular}

Table 2: Oracle-Automated Verification for the LoRaWAN 1.1 Join w.r.t. $E D$ and $A S$ : Summative Results

In this setting, our analysis shows that sync_ED_AS fails. Further, confusion-freeness CF_ED_AS_packet_key fails, even in the main variant of the protocol where the $A S$ obtains the AppSKey from the NS (see Subsection 2.1). In this case, our falsifying traces shows that the attacker can cross-wire device addresses and the corresponding AppSKeys as well inject wrong application data-packets.

The second row of Table 2 shows the $\mathcal{M}_{\text {NS-weakCorrupt }}$ model, in which the channel between $N S$ and $J S$ is considered to be insecure. In this case, the aforementioned properties (sync_ED_AS, CF_ED_AS_packet_key) continue to fail. Moreover, a stronger 3-party variation of the confusion-freeness property, CF_ED_JS_AS_key, fails (also due to the hierarchical implication). More specifically, CF_ED_JS_AS_key fails in the $\mathcal{M}_{\text {NS-weakCorrupt }}$ model because the attacker can confuse not only the $A S$, but also the $J S$ as to which devices sent which JoinRequests or messages. Concretely, the trace found here shows that an attacker who controls the channel between $N S$ and $J S$ never forwards anything to the $J S$, and swaps two devices' requests on the $N S-A S$ channel.

Finally, the third row of Table 2 shows that if all channels are secure (i.e., in the $\mathcal{M}_{\mathrm{AS}-\mathrm{NS}-\mathrm{Secure}}$ ), all these 2-party and 3-party properties hold.

\subsection{Implications of the Attacks Found}

The trace corresponding to CF_ED_AS_packet_key failing in the Desync Model shows that $A S$ can be (adversarially) confused when considering from which devices application-layer messages have come. As a result, $A S$ may be ultimately unable to decode a message from some $E D_{1}$, because they have received some AppSKey 2 to link to $E D_{1}$ when this key instead corresponds to some $E D_{2}$.

It is not clear how the $A S$ would behave in this case, or if it would store the wrong address for $E D_{1}$ for a period of time.

With sync_ED_AS failing, and with the specs not being clear on how the $A S$ needs to respond, it also means that an $E D$ may well believe it is sending LoRa data to an $A S$, when it actually is not. In fact, a malicious party in between the $N S$ and $A S$ gets hold of this (encrypted) data. This creates not only data loss for the ED's owner, but also data-collection by malicious parties, with no hope that the $A S$ would track or signal any malfunction.

Countermeasures. To stop the failure of sync_ED_AS and CF_ED_AS_packet_key, we first and foremost recommend that the LoRaWAN 1.1 explicitly require that the $A S$ check the format of the first data-packet served and end in "error" if the decryption of this eventually fails. Moreover, it should not store any long-term data with respect to this failed message.
Furthermore, we observe that the AKE properties that fail in the $\mathcal{M}_{\mathrm{LoRa} 1.1 \mathrm{Spec}}$ model hold in the model $\mathcal{M}_{\mathrm{AS} \text {-NS-Secure. }}$ In practice, this means that strengthening the LoRaWAN specification's requirements to have a secure channel between the $N S$ and the $A S$ can fix the aforementioned AKE/LoRa 1.1 failing. However, the security of this channel may often fall under the management of the $N S$ and $A S$. This means that even if the LoRaWAN specifications were to require that these channels be secure, we cannot be sure that this would be attained in practice.

Our analysis in the $\mathcal{M}_{\mathrm{NS} \text {-weakCorrupt setting tells us }}$ that any loosening of the trust in $N S$ results in several keyagreement properties failing. Notably, because confusion freeness fails, it means that we cannot guarantee that an $A S$ will have a correct view as to which device it is speaking to. At the application-level, this is a significant attack.

Due to the aforementioned issues with security ownership and risk, a more costly countermeasure that may prevent the failure of sync_ED_AS and CF_ED_AS_packet_key is the possible introduction of a clear key-confirmation step between the $A S$ and $E D$. Or, even further, one can consider making the $A S$ part of the Join and not having the AppSKey delivered "blindly" to it alongside the first data-packet

Overall, it maybe be advisable that the Join Procedure be redesigned such that the $A S$ cannot be confused irrespective of the security of these channels and trust in $N S$. However, it is important that any modifications should remain as close as possible to the current Join design.

\section{A New, Secure LoRaWAN 1.1 Join}

We now propose an amended version of the LoRaWAN 1.1 Join, which we call LORA $3-A K A^{+}$.

This new design is rooted in the analysis results presented in Subsection 4.6.

In order to prevent the $A S$ from becoming confused as to which device it is communicating with, the $N S$ provisioner must ensure the security of the incoming and outgoing channels. However, it would be interesting if we could attain the same level of security by instead placing less trust in the messages forwarded by the Network Server. Indeed, it would be ideal if we could propose a design that is secure even when these NS-originating channels are untrusted or compromised. To this end, we leverage that -unlike in the LoRaWAN 1.1 Join- the $A S$ could be an active part of the Join. Precisely, in LoRA $3-\mathrm{AKA}^{+}$, we augment the LoRaWAN 1.1 Join such that the $A S$ is minimally active. Arguably, this is as backwards-compatible as possible with the current LoRaWAN 1.1 Join.

So, at the cost of a small increase in latency, we attain the LORA $3-\mathrm{AKA}^{+}$Join, which is a secure multi- 


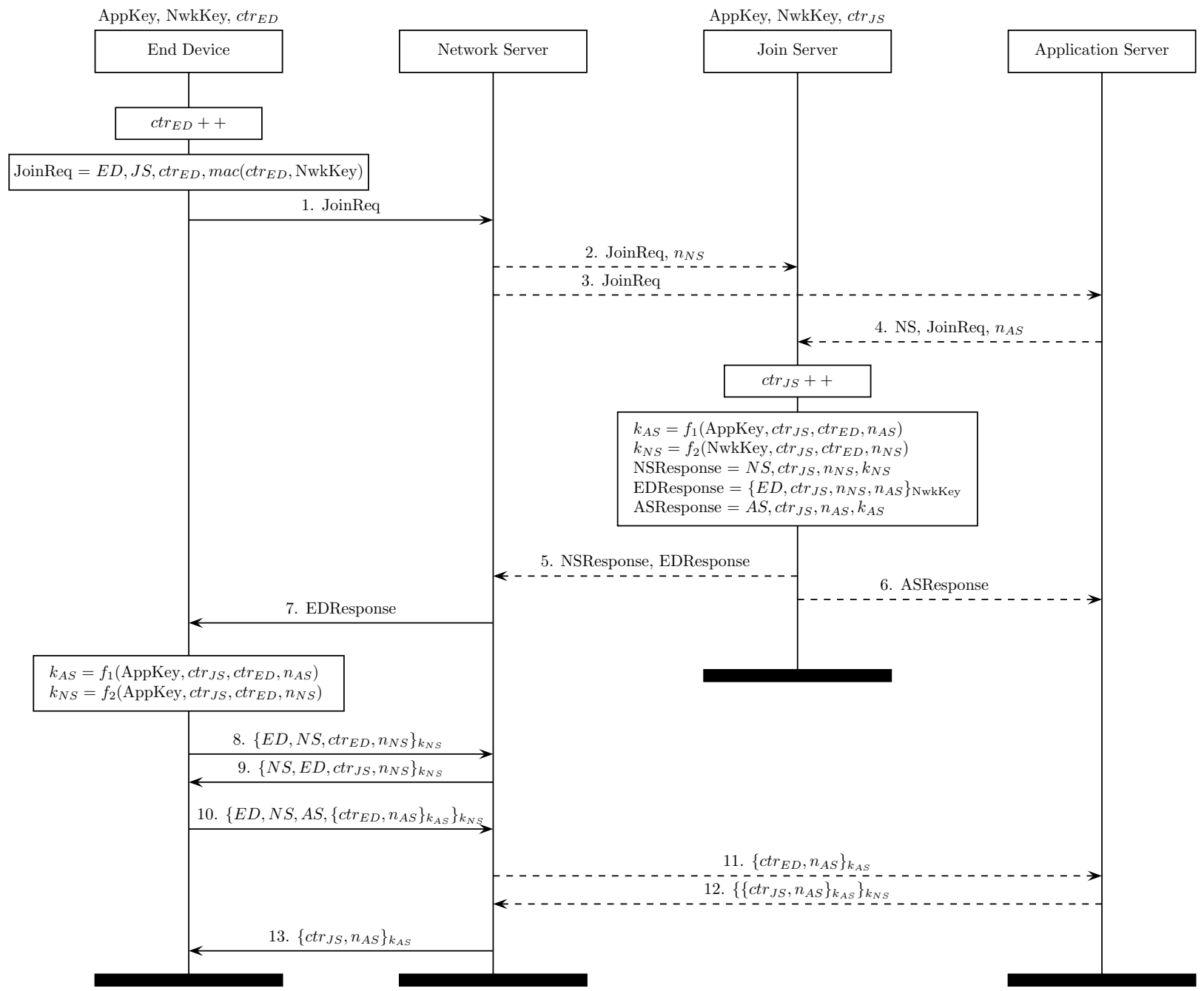

Figure 5: LoRA 3-AKA ${ }^{+}$- A Minimally-Augmented LoRaWAN 1.1. Join with More Security, Less Trust. Secure channels are displayed with dashed lines " $--\rightarrow$ ".

party AKE, even when the channels out of the $N S$ are compromised.

Essential Features of LORA 3-AKA ${ }^{+}$Compared to the LoRaWAN 1.1 Join. We work with the following principles in mind:

- The parties and network topology remain the same as in LoRaWAN 1.1.

- The Application Server is "minimally" active during the Join Procedure: it contributes a nonce to the Join Request, adding randomness to the encryption key AppSKey and the MAC key NwkSKey.

- We envisage that the LoRA $3-\mathrm{AKA}^{+}$specification require (with a 'MUST' keyword) that the $N S-A S$ and $N S$-JS channels be secure; however, we show that even if these are compromised the (confusionfreeness) security guarantees are not lost.

- A "Key Confirmation" step is added at the end of the protocol, whereby the involved parties check that they have mutually agree on the established session keys. This key confirmation step occurs before the record phase begins.

The LORA $3-\mathrm{AKA}^{+}$design is as close as possible to LoRaWAN 1.1 Join, whilst giving protection to the Application Server against any possible (key/device) con- fusion attacks such as those we have highlighted against LoRaWAN 1.1.

The LORA 3-AKA ${ }^{+}$Protocol. Figure 5 gives an overview of LORA $3-\mathrm{AKA}^{+}$

The protocol LoRA $3-\mathrm{AKA}^{+}$is split into two main phases. First, the keys are established using a process very similar to LoRaWAN 1.1. Then, a set of "Key Confirmation" messages are sent between the End Device and the Network Server and Application Server respectively, in which the newly generated keys are checked for authenticity.

For readability, in this description we do not include references to parties' names or configuration parameters. We also collapse the multiple keys NwkSKey, FNwkSIntKey, SNwkSIntKey into a single value $\left(k_{N S}\right)$. However, our Tamarin models do model the addition of parties' names and all LoRaWAN keys.

The LORA $3-\mathrm{AKA}^{+}$protocol proceeds as follows:

1) The End Device $E D$ generates a Join Request in the same way as in LoRaWAN 1.1, incrementing its DevNonce counter and sending it along with the request command, including a MAC of the message using the NwkKey. 
2-3) The Network Server $N S$ forwards the Join Request to the Join Server, adding a freshly generated nonce NwkNonce. The Join Request is also forwarded as-is to the Application Server.

4) The Application Server $A S$ acknowledges the Join Request by forwarding another copy of it to the Join Server, adding a freshly generated nonce AppNonce.

5-7) The Join Server processes the Join Request, generating the following values:

$k_{A S}=f_{1}$ (NwkKey, JoinNonce, DevNonce, AppNonce)

$k_{N S}=f_{2}$ (NwkKey, JoinNonce, DevNonce, NwkNonce)

NSResponse $=$ JoinNonce, NwkNonce, $k_{N S}$

EDResponse $=\{\text { JoinNonce, NwkNonce, AppNonce }\}_{\text {NwkKey }}$

ASResponse $=$ JoinNonce, AppNonce, $k_{A S}$,

NSResponse, ASResponse and EDResponse are then sent to $N S, A S$ and $E D$ (via $N S$ ), respectively.

8) The End Device re-calculates the keys independently. It then sends the first key confirmation request to the Network Server, consisting of the values DevNonceand NwkNonce, encrypted with the calculated $k_{N S}$.

9) The Network Server responds with a key response message, containing JoinNonce and NwkNonce.

10-13) The End Device sends a second key-confirmation message, this time to the Application Server (via the Network Server). These messages are encrypted using $k_{A S}$, making the message-body unreadable to the Network Server. The key-confirmation message contains values DevNonce and AppNonce, and the keyconfirmation response contains values JoinNonce and AppNonce.

\subsection{Analysis of the LORA $3-\mathrm{AKA}^{+}$Protocol.}

We modelled the LORA $3-\mathrm{AKA}^{+}$protocol in the $\mathcal{M}_{\text {AS-NS-Secure }}$ and $\mathcal{M}_{\mathrm{NS} \text {-weakCorrupt }}$ models. These models represent the best-case and worst-case scenarios, depending on whether or not the $N S$ is part of the fully trusted environment alongside the $J S$ and $A S$.

We modelled the same main properties as for LoRaWAN 1.1 Join.

(1) weak agreement between $E D$ and $A S$ (wa_ED_AS)

(2) two-party (CF_ED_AS_packet_key)

(3) explicit three-party (CF_ED_JS_AS_key) confusion-freeness confusion-freeness

Our analysis inside Tamarin proves that wa_ED_AS, CF_ED_AS_packet_key, CF_ED_JS_AS_key, all hold on LORA $3-\mathrm{AKA}^{+}$, in both the $\overline{\mathcal{M}}_{\mathrm{AS}-\mathrm{NS}-\text { Secure }}$ and $\mathcal{M}_{\text {NS-weakCorrupt }}$ threat models. In particular, even when the channels in and out of the $N S$ were compromised, the fact that the $A S$ is alive, as well as the addition of the key confirmation step, prevents any agreement insecurities inside LORA $3-\mathrm{AKA}^{+}$.

Intuitively, although a corrupt $N S$ is able to grant the adversary access to the secure channels and thus read, redirect or inject messages, the addition of explicit checks prevents risk of confusion. Since the confirmation check between the $E D$ and $A S$ is encrypted using the justestablished key (which the adversary cannot reproduce without knowledge of the long term AppKey), agreement and aliveness is ensured.

\section{Verification Details}

In this section, we join together our security analysis results, and present certain details of the verification process of the LoRaWAN 1.0, LoRaWAN 1.1 Join and the LORA $3-\mathrm{AKA}^{+}$Join protocol that we eluded to in Section 4 and Section 5.

Models. We produced 15 Tamarin models altogether: one for LoRaWAN 1.0, twelve for the different LoRaWAN 1.1 scenarios and two for LORA $3-\mathrm{AKA}^{+}$. Most Tamarin models contain around 1300 lines of code.

We also recall here our different threat models:

- in $\mathcal{M}_{\text {LoRa1.1Spec }}$, the channel between the $N S$ and the $J S$ is secure, but the channel between the $N S$ and $A S$ is insecure.

- in $\mathcal{M}_{\mathrm{AS}-\mathrm{NS}-\mathrm{Secure}}$, the channel between the $N S$ and the $J S$ is secure, and the channel between the $N S$ and $A S$ is secure (i.e., it tightens the $\mathcal{M}_{\text {LoRa1.1Spec }}$ threat-model).

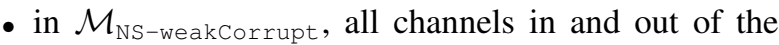
$N S$ are insecure but the $N S$ does follow the protocol (i.e., it moderately loosens the level of trust that the LoRaWAN specification places on the $N S$ ).

Due to this faithfulness and thus the resulting complexity of our modelling (see Sections 4 and Section 5), the standard heuristics employed by the Tamarin prover to prove the models' properties do not reliably result in termination. Consequently, the proofs of all properties required the assistance of a proof oracle (see Section 2, on Tamarin oracles). These oracles were constructed by using the web interface of the Tamarin prover and stepping through the proofs of the desired property. The steps taken in this way, for each model, were then encoded into a corresponding Python script of the order of between 100 and 150 lines of code. Using these oracles, our lemmas are then proved automatically, without any further intervention.

All the models as well as any oracles, and the saved versions of any manual proofs are available at [16], together with instructions on how to run them.

Summary of Results. Table 3, Table 4 and Table 5 summarise all the results we have discussed so far (and a few more), for LoRaWAN 1.0, LoRaWAN 1.1 and LoRA $3-\mathrm{AKA}^{+}$, respectively. A $\checkmark$ in the table indicates that the property holds in that model, a $\boldsymbol{X}$ indicates that the TAMARIN PROVER found a trace that violated the property. Further, an "NA" entry indicates that the property does not apply to that model, while "NM" indicates that the property was not modelled. Some of the security goals presented in Subsection 4.4 are omitted, because their validity is immediately deducible from the results of other properties, following from our hierarchy of goals presented in Subsection 4.5. Finally, "NT" means that the proof did not terminate given our current oracles. It is possible that with re-working of the associated oracle these would terminate, but this is uncertain due to the underlying undecidability of the verification problem at hand. 


\begin{tabular}{|c|c|c|c|c|c|c|c|c|c|c|c|c|}
\hline Threat Model & \multicolumn{4}{|c|}{$\mathcal{M}_{\text {AS-NS-Secure }}$} & \multicolumn{4}{|c|}{$\mathcal{M}_{\text {LoRa1.1Spec }}$} & \multicolumn{4}{|c|}{$\mathcal{M}_{\text {NS-weakCorrupt }}$} \\
\hline Synchronisation & \multicolumn{2}{|c|}{ Sync } & \multicolumn{2}{|c|}{ Desync } & \multicolumn{2}{|c|}{ Sync } & \multicolumn{2}{|c|}{ Desync } & \multicolumn{2}{|c|}{ Sync } & \multicolumn{2}{|c|}{ Desync } \\
\hline Key Delivery & $\begin{array}{l}\text { NS key } \\
\text { deliv. }\end{array}$ & $\begin{array}{l}\text { JS key } \\
\text { deliv. }\end{array}$ & $\begin{array}{c}\text { NS key } \\
\text { deliv. }\end{array}$ & $\begin{array}{c}\text { JS key } \\
\text { deliv. }\end{array}$ & $\begin{array}{c}\text { NS key } \\
\text { deliv. }\end{array}$ & $\begin{array}{c}\text { JS key } \\
\text { deliv. }\end{array}$ & $\begin{array}{c}\text { NS key } \\
\text { deliv. }\end{array}$ & $\begin{array}{l}\text { JS key } \\
\text { deliv. }\end{array}$ & $\begin{array}{c}\text { NS key } \\
\text { deliv. }\end{array}$ & $\begin{array}{l}\text { JS key } \\
\text { deliv. }\end{array}$ & $\begin{array}{c}\text { NS key } \\
\text { deliv. }\end{array}$ & $\begin{array}{c}\text { JS key } \\
\text { deliv. }\end{array}$ \\
\hline \multicolumn{13}{|l|}{ Security Goal } \\
\hline key_secrecy & $\checkmark$ & $\checkmark$ & $\checkmark$ & $\checkmark$ & $\checkmark$ & $\checkmark$ & $\checkmark$ & $\checkmark$ & $\checkmark$ & $\checkmark$ & $\checkmark$ & $\checkmark$ \\
\hline wa_ED_JS & $\checkmark$ & $\checkmark$ & $\checkmark$ & $\checkmark$ & $\checkmark$ & $\checkmark$ & $\checkmark$ & $\checkmark$ & $\checkmark$ & $\checkmark$ & $\checkmark$ & $\checkmark$ \\
\hline wa_ED_AS & $\checkmark$ & $\checkmark$ & $\checkmark$ & $\checkmark$ & $\checkmark$ & $\checkmark$ & $\checkmark$ & $\checkmark$ & $x$ & $x$ & $x$ & $x$ \\
\hline nia_ED_JS & $\checkmark$ & $\checkmark$ & $\sqrt{ }$ & $\checkmark$ & $\checkmark$ & $\sqrt{ }$ & $\sqrt{ }$ & $\sqrt{ }$ & $x$ & $x$ & $x$ & $x$ \\
\hline sync_ED_AS & $\checkmark$ & $\checkmark$ & $\checkmark$ & $\checkmark$ & $\checkmark$ & $\checkmark$ & $x$ & $x$ & NM & NM & $x$ & $x$ \\
\hline CF_ED_ASS_packet_key & $\checkmark$ & $\checkmark$ & $\checkmark$ & $\checkmark$ & NT & NT & $x$ & $x$ & $x$ & $x$ & $\checkmark$ & $\checkmark$ \\
\hline
\end{tabular}

Table 3: Main Verification Results for the LoRa 1.1 Join

\begin{tabular}{|c|c|}
\hline Security Goal & $\mathcal{M}_{\text {LoRaSpec }}$ \\
\hline \hline key_secrecy & $\checkmark$ \\
\hline sync_ED_NS & $\boldsymbol{X}$ \\
\hline wa_ED_NS & $\checkmark$ \\
\hline nia_ED_NS & $\boldsymbol{x}$ \\
\hline ia_ED_NS & $\boldsymbol{x}$ \\
\hline replay resistance & $\boldsymbol{x}$ \\
\hline
\end{tabular}

Table 4: LoRa 1.0 Results

\begin{tabular}{|r|c|c|c|}
\hline Threat Model & $\mathcal{M}_{\text {AS-NS-Secure }}$ & $\mathcal{M}_{\text {LoRa1.1Spec }}$ & $\mathcal{M}_{\text {NS-weakcorrupt }}$ \\
\hline \hline \multicolumn{5}{|l}{} & & \\
\hline \hline Security Goal & $\checkmark$ & NA & $\checkmark$ \\
\hline key_secrecy & $\checkmark$ & NA & $\checkmark$ \\
\hline wa_ED_JS & $\checkmark$ & NA & $\checkmark$ \\
\hline wa_ED_AS & $\checkmark$ & NA & $\checkmark$ \\
\hline nia_ED_JS & $\checkmark$ & NA & $\checkmark$ \\
\hline ia_ED_JS & $\checkmark$ & NA & $\checkmark$ \\
\hline sync_ED_AS & $\checkmark$ &
\end{tabular}

Table 5: Main Verification Results for LoRA 3-AKA ${ }^{+}$
Concerning LoRaWAN 1.0, we recount the main results:

1) We re-prove that synchronisation between $E D$ and $N S$ (i.e., the property defined as sync_ED_NS in Section 4) fails. This was known from arguably sketchy modelling of LoRaWAN1.0 in [4];

2) We show non-injective agreement and injective agreement between $E D$ and $J S$ also fails;

3) By modelling the small domain of nonces, we show that replay resistance fails. This is the first symbolic-verification analysis finding this attack, known since [1].

For LoRaWAN 1.1, we re-summarise the main results:

1) In the $\mathcal{M}_{\text {LoRa1.1Spec }}$ threat-profile, we have the following main results:

a) using our "Desync Model", in the case where the $N S$ delivers the AppSKey to the $A S$, as well as in the case where the $J S$ does so, we formally prove that the $A S$ and the $E D$ can be desynchronised (i.e., sync_ED_AS and CF_ED_AS_packet_key failing). The first trace found (on sync_ED_AS failing) shows that that $A S$ cannot know where a packet originates from. The second trace found is synonymous: there are two genuine device addresses with two AppSKeys and two encrypted data packets present in the system, and the attacker inflicts a message onto $A S$ with the first device address, the second AppSKey and a fraudulent data packet, yet the $A S$ "throws" no error.

2) In the $\mathcal{M}_{\mathrm{AS}-\mathrm{NS} \text {-Secure }}$ threat-profile, we prove that the insecurity problems found in $\mathcal{M}_{\mathrm{LoRa} 1.1 \mathrm{Spec}}$ threat-profile disappear.

3) In the $\mathcal{M}_{\mathrm{NS} \text {-weakCorrupt }}$ threat-profile then we show that several agreement properties fail, as expected.

4) For LoRA $3-\mathrm{AKA}^{+}$, we show that not only it is AKE-secure in the $\mathcal{M}_{\text {LoRa1.1Spec }}$ model, but it maintains AKE-security even in the 4-party sense (i.e., w.r.t. CF_ED_JS_AS_key holding), even in the $\mathcal{M}_{\mathrm{NS} \text {-weakCorrupt }}$ model.
Other Statistics: Timing, Proof-steps. All models were executed using the Tamarin prover running on a server with 2 Intel Xeon E5-2667 CPUs (16 cores, 32 threads) and $378 \mathrm{~GB}$ of RAM.

The average time over all automatic proofs in the $\mathcal{M}_{\text {LoRa1.1Spec }}$ model, which is the one capturing the LoRaWAN specifications as closely as possible, is around 10 minutes using 10 cores.

\section{Engagement with the LoRa Alliance}

As part of the research process, we engaged with the LoRa Alliance. As well as performing responsible disclosure of our findings, we also held a series of interactions with respect to the wider scope of formally verifying the current, as well as future, LoRaWAN specifications.

In relation our findings, the Security Working Group (SWG) and the Technical Committee (TC) of the LoRa Alliance are in alignment with us regarding the following main aspects:

1) clear requirements ought to be stipulated with respect to the security (i.e., confidentiality and integrity) of the NS-AS channel;

2) the $A S$ should, to some extent, become an active part of Join, as this would increase the capabilities of the $A S$ to verify the authenticity of session keys and application-layer messages.

During this interaction, we confirmed that the LoRaWAN specifications do not explicitly require that the $N S-A S$ channel be secure.In general, requirements on the $N S-A S$ channel and the $A S$ itself are slightly underspecified in the current version of the specifications due to the fact that the $N S$ and the $A S$ have been initially thought of as co-located parties. As the commissioning of proprietary Application Servers becomes increasingly common, the LoRa Alliance acknowledges that the issues of the under-specification of the security of the $N S-A S$ channel as well the amount of trust placed on the $N S$ 
have become more acute. This confirms the validity of our $\mathcal{M}_{\text {LoRa1.1Spec }}$ and $\mathcal{M}_{\mathrm{NS} \text {-weakCorrupt }}$ models.

Indeed, within the LoRa Alliance SWG, work is already ongoing to properly specify requirements on the $N S-A S$ channel. With regards to the trust placed in the $N S$, it is to be noted that parts of our LORA $3-\mathrm{AKA}^{+}$ design were generally welcome. Concretely, the $A S$ contributing with a nonce to the Join procedure is being considered by the LoRa Alliance SWG as a possible solution going forward. However, the key-confirmation step is viewed as potentially too computationally demanding on the end devices; as such, the latter could be simplified and/or made optional, if ever to be adopted. Along these lines, we will be working with the LoRa Alliance SWG and the LoRa Alliance TC (of which we have become a part), to formally verify iterations of new specifications of the LoRaWAN Join that fit our recommendations and are agreeable with other requirements (e.g., $A S$-related connectivity, energy-consumption of the end devices, etc.).

Last but not least, we note that during our latest discussion with the LoRa Alliance (which occurred after the acceptance for publication of this manuscript), it transpired that there is a discrepancy between the LoRaWAN 1.1 specifications and our models. We believe this gap is worth mentioning; this is as follows. In the specifications, it is the $N S$ which generates the DevAddr used by the $E D$ in the application-layer packets, whereas in our models ${ }^{11}$, the $J S$ generates the DevAddr and the consequent mapping between DevEUIs and DevAddrs. For future work that extends our models in this setting, new properties hinging more strongly on agreement properties with the $N S$ would arguably require verification. Also, more generally, note that the trust on the $N S$ would be increased in this setting, and as such our $\mathcal{M}_{\mathrm{NS} \text {-weakcorrupt }}$ model may require rethinking.

\section{Conclusions}

Here we briefly summarise our results and discuss options for future avenues of research.

Main Recommendations for LoRaWAN 1.0. Although this is linked to an older recommendation from [1], our results formally prove that the domain of the nonces in 1.0 ought to be increased. The LoRa Alliance looked to repair the replay resistance failure by creating a counterbased mechanism for nonces. Whilst we do not prove a direct attack on this, we believe that a better approach would be to increase the domain of the nonces, which would prevent the replay resistance failure, without introducing additional risks.

Main Recommendations for LoRaWAN 1.1. Firstly, our attacks show that the LoRa Alliance should explicitly demand the $N S$ and $A S$ channel be secured. That is, for instance, the trace that shows CF_ED_AS_packet_key fail in the "Desync" model would not be possible if the attacker could not inflict his own data-packet in between the $N S$ and $A S$, or swap the device address in that same message.

11. The confusion on our part stems from the fact that in the 1.1 specification the actual " $J S$ " and " $N S$ " words are often confused, due to incremental modifications from LoRaWAN 1.0 specifications to LoRaWAN 1.1 specifications - in which the $J S$ is introduced.
Secondly, linked to the above, we believe that a tightening of the specification around how the $A S$ is to handle errors upon failure to decode a message is necessary. As per the above, confusion attacks were shown to be possible (i.e. when $A S$ is served a device address and an AppSKey that do not match). If a packet does not decode correctly, it is not clear how the $A S$ should continue.

Thirdly, we show that relying on one single crux of trust (i.e., the current $N S$ in LoRaWAN 1.1) is dangerous. Based on the current LoRaWAN specifications, if only the $N S$-to- JS channel becomes compromised, then we show that several AKE security goals can fail.

Results Discussion. We have constructed multiple models of the LoRaWAN Join procedure, We have shown that the LoRaWAN 1.1 Join protocol, while presenting improvements over 1.02 , is still secure only under an adversary model weaker than that given in the specifications. We introduced a new security goal, described as confusion freeness, which models a form of indirect agreement between the End Device and Application Server.

The core of these problems arises from two issues with the LoRaWAN join procedure. First, the Network Server, which acts as a forwarding agent, is implicitly trusted. Second, the Application Server is not active during the Join procedure. Even with the Join Server being a root of trust, the Application Server must also have confidence that $N S$ correctly forwards the results of the procedure. To remedy this, we proposed and verified a new protocol, LORA 3-AKA ${ }^{+}$, which addresses these concerns, and satisfies security goals even against our most powerful adversary.

As we continue to engage with the LoRa Alliance, at http://loraverif.github.io, we will maintain a longer version of this paper that contains further details, and a log of updates to our Tamarin models.

Future Work. Even if this is not in the LoRaWAN specifications, we believe strong device authentication and device unlinkability should be studied. The risk of versiondowngrade attacks potentially also exists in LoRaWAN. Modelling such attacks may be useful to get a more complete view of the LoRaWAN ecosystem.

Acknowledgements: The research was partly funded by the following EPSRC projects: "Improving customer experience while ensuring data privacy for intelligent mobility - EP/N028295/1" and "AutoPaSS: Automatic Verification of Complex Privacy Requirements in UnboundedSize Secure Systems - EP/S024565/1"

\section{References}

[1] G. Avoine and L. Ferreira. Rescuing LoRaWAN 1.0. In IACR Cryptology ePrint Archive, 2017.

[2] K. Bhargavan, I. Boureanu, A. Delignat-Lavaud, P. Fouque, and C. Onete. A formal treatment of accountable proxying over TLS. In 2018 IEEE Symposium on Security and Privacy, SP 2018 , Proceedings, 21-23 May 2018, San Francisco, California, USA, pages 799-816, 2018.

[3] B. Blanchet. Security protocol verification: Symbolic and computational models. In P. Degano and J. D. Guttman, editors, Principles of Security and Trust, pages 3-29, Berlin, Heidelberg, 2012. Springer Berlin Heidelberg.

[4] I. Butun, N. Pereira, and M. Gidlund. Analysis of LoRaWAN v1.1 security: research paper. pages 1-6, 062018. 
[5] S. Canard and L. Ferreira. Extended 3-Party ACCE and Application to LoRaWAN 1.1. In J. Buchmann, A. Nitaj, and T. Rachidi, editors, Progress in Cryptology - AFRICACRYPT 2019, pages 2138, Cham, 2019. Springer International Publishing.

[6] C. Cremers, M. Horvat, J. Hoyland, S. Scott, and T. van der Merwe. A comprehensive symbolic analysis of TLS 1.3. In Proceedings of the 2017 ACM SIGSAC Conference on Computer and Communications Security, CCS '17, pages 1773-1788, New York, NY, USA, 2017. ACM.

[7] D. Dolev and A. Yao. On the Security of Public-Key Protocols. IEEE Transactionson Information Theory 29, 29(2):198-208, 1983.

[8] N. Durgin, P. Lincoln, J. Mitchell, and A. Scedrov. Undecidability of Bounded Security Protocols. In E. Clarke and N. Heintze, editors, In Proceedings of Workshop on Formal Methods and Security Protocols (FMSP'99), Trento, Italy, 1999. ACM New York.

[9] M. Eldefrawy, I. Butun, N. Pereira, and M. Gidlund. Forma security analysis of LoRaWAN. Computer Networks, 148:328 339, 2019.

[10] G. Lowe. A Hierarchy of Authentication Specifications. In S. Foley and J. Millen, editors, In Proceedings of the 10th IEEE workshop on Computer Security Foundations (CSFW'97), pages 31-43, Massachusetts, US, 1997. IEEE Computer Society.

[11] G. Lowe. A hierarchy of authentication specifications. pages 3143. IEEE Computer Society Press, 1997

[12] S. Meier, B. Schmidt, C. Cremers, and D. Basin. The TAMARIN Prover for the symbolic analysis of security protocols. In N. Sharygina and H. Veith, editors, Computer Aided Verification, pages 696701, Berlin, Heidelberg, 2013. Springer Berlin Heidelberg.

[13] N. Sornin. LoRaWAN 1.1 Specification (April 2018). LoRa Alliance, $\quad$ https://lora-alliance.org/sites/default/files/2018-04/ lorawantm_specification_-v1.1.pdf, 1 .

[14] N. Sornin, M. Luis, T. Eirich, T. Kramp, and O. Hersent. LoRaWAN Specification, Version V1.0.2;. LoRa Alliance, https: //lora-alliance.org/resource-hub/lorawanr-specification-v102.

[15] The Tamarin Team. Tamarin prover manual, 2016. https: //tamarin-prover.github.io/manual/tex/tamarin-manual.pdf [Online: accessed 09-April-2019].

[16] S. Wesemeyer, I. Boureanu, Z. Smith, and H. Treharne. LoRaWAN models. GitHub, 2020. https://github.com/ lorawan-tamarin-analysis/lorawan-tamarin-analysis.

\section{Appendix A. \\ Tamarin Model Snippets}

We give code snippets from our Tamarin prover implementations. Our full source code is available at https://github.com/lorawan-tamarin-analysis/ lorawan-tamarin-analysis.

LoRaWAN 1.0 Nonce Selection shows our approach to generating nonces with the potential to repeat (due to the short length) within the Join procedure.

Confusion Freeness shows our definition of the Confusion Freeness security property, a specialised indirect agreement property.

Rule Example shows an example of one of our rules. These rules capture in-depth details, modelling each of the individual keys generated and referencing specific lines of the LoRaWAN specification used in creating our model.

Modelling Configurations shows how we use simple rules, early on in the specification, to easily modify our different sets of assumptions, such as the use of secure channels or how the Application Server receives AppSKey.

\section{Appendix B. Expanded Diagrams}

We include some enlarged versions of the message sequence diagrams included within the paper. We also include additional details, such as agent names included in messages. Such terms have little impact on our security analysis, but more closely represent a real implementation. Figures 10 and 11 contain the LoRaWAN 1.1 protocol, showing the two different methods of AppSKeydelivery. In all diagrams, secure channels are displayed with dashed lines $-\rightarrow \rightarrow$. 


\section{Figure 6: LoRaWAN 1.0 Parameters Example}

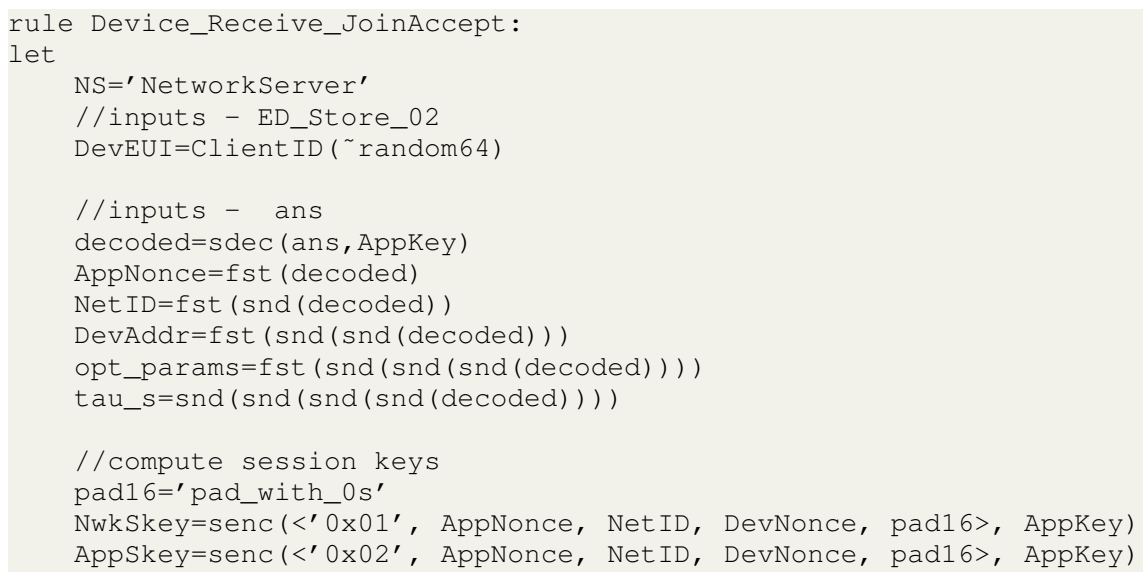

Figure 7: LoRaWAN 1.0 Nonce Selection

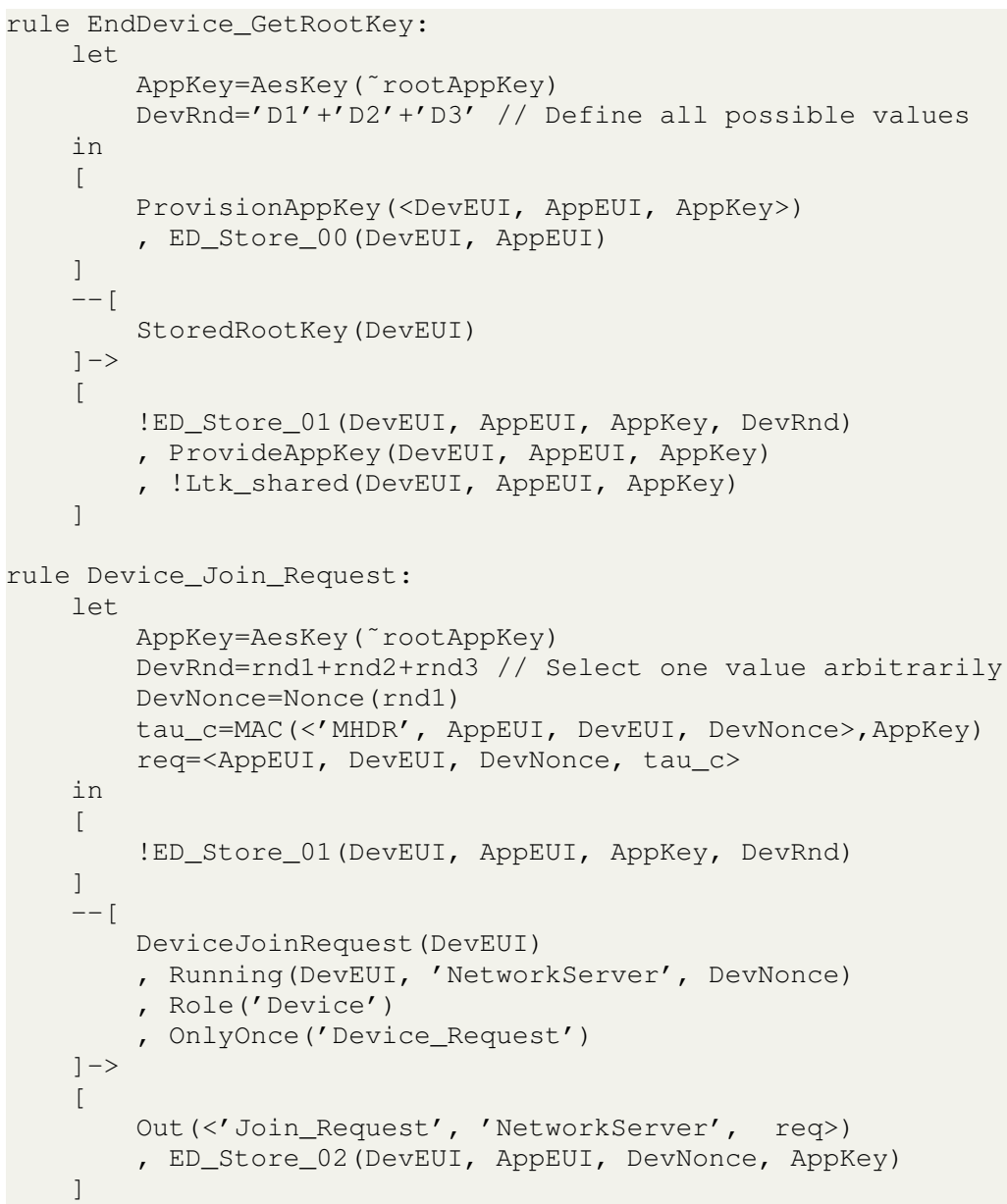


Figure 8: Confusion Freeness

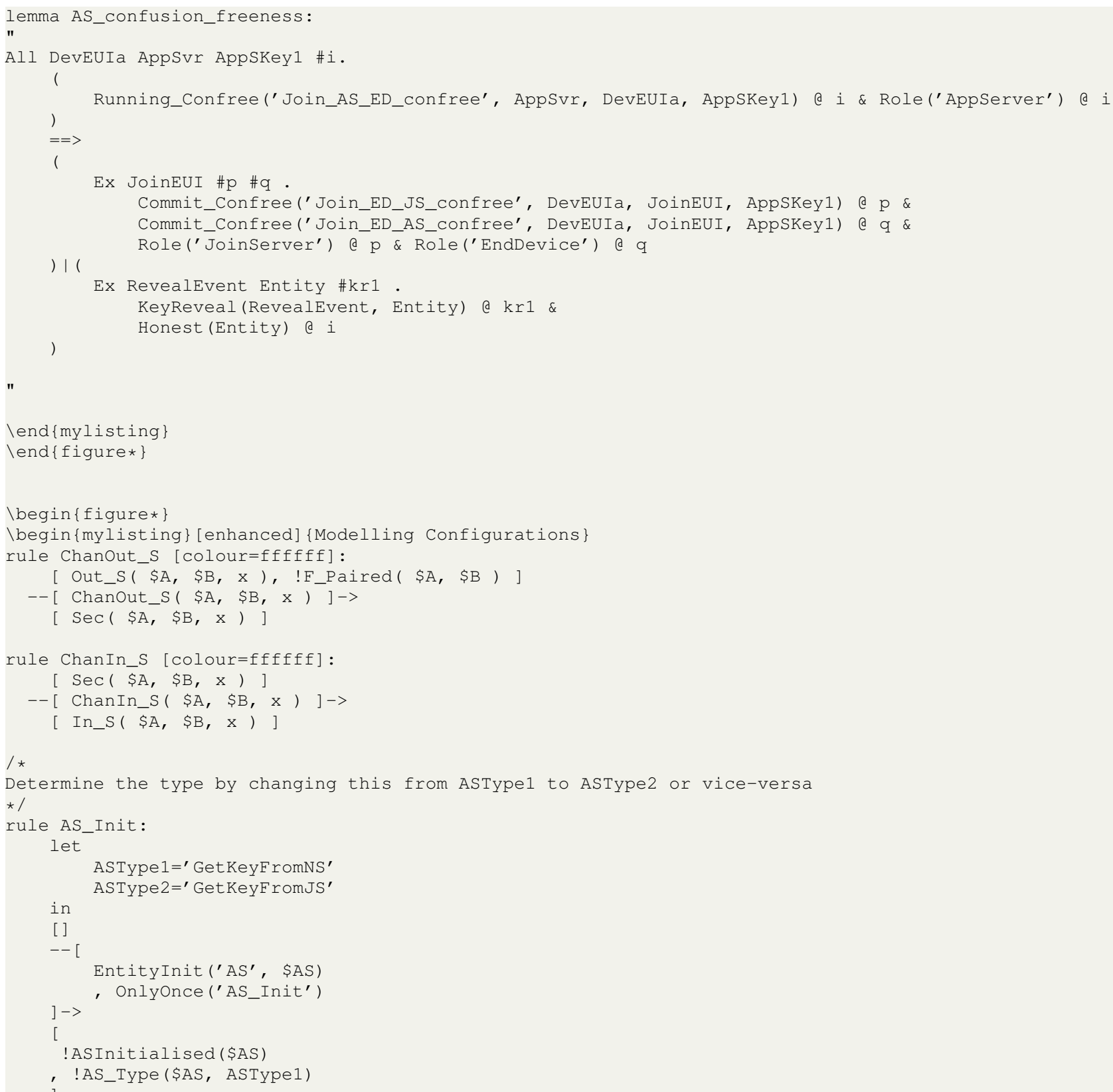




\section{Figure 9: Rule Example}

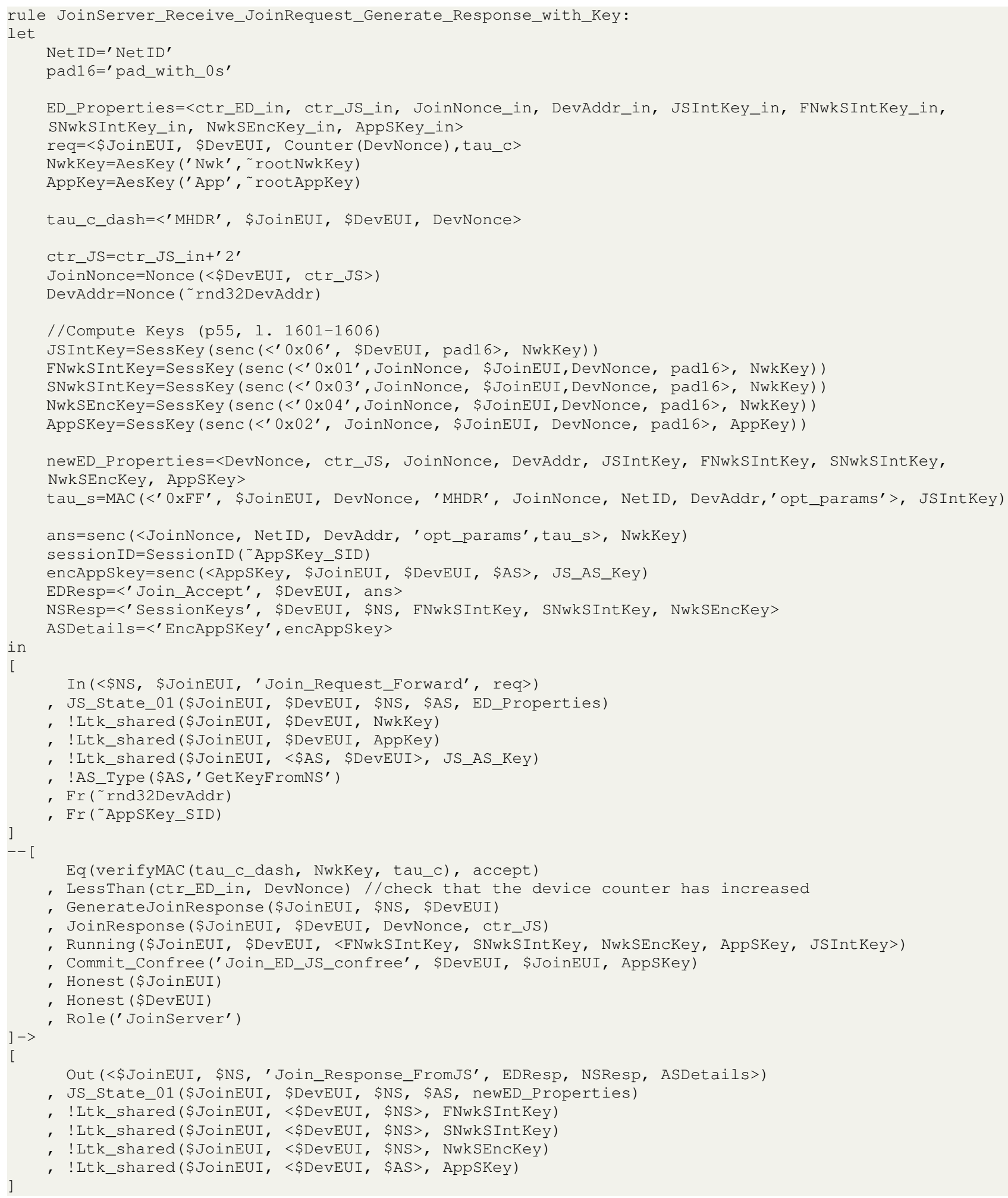




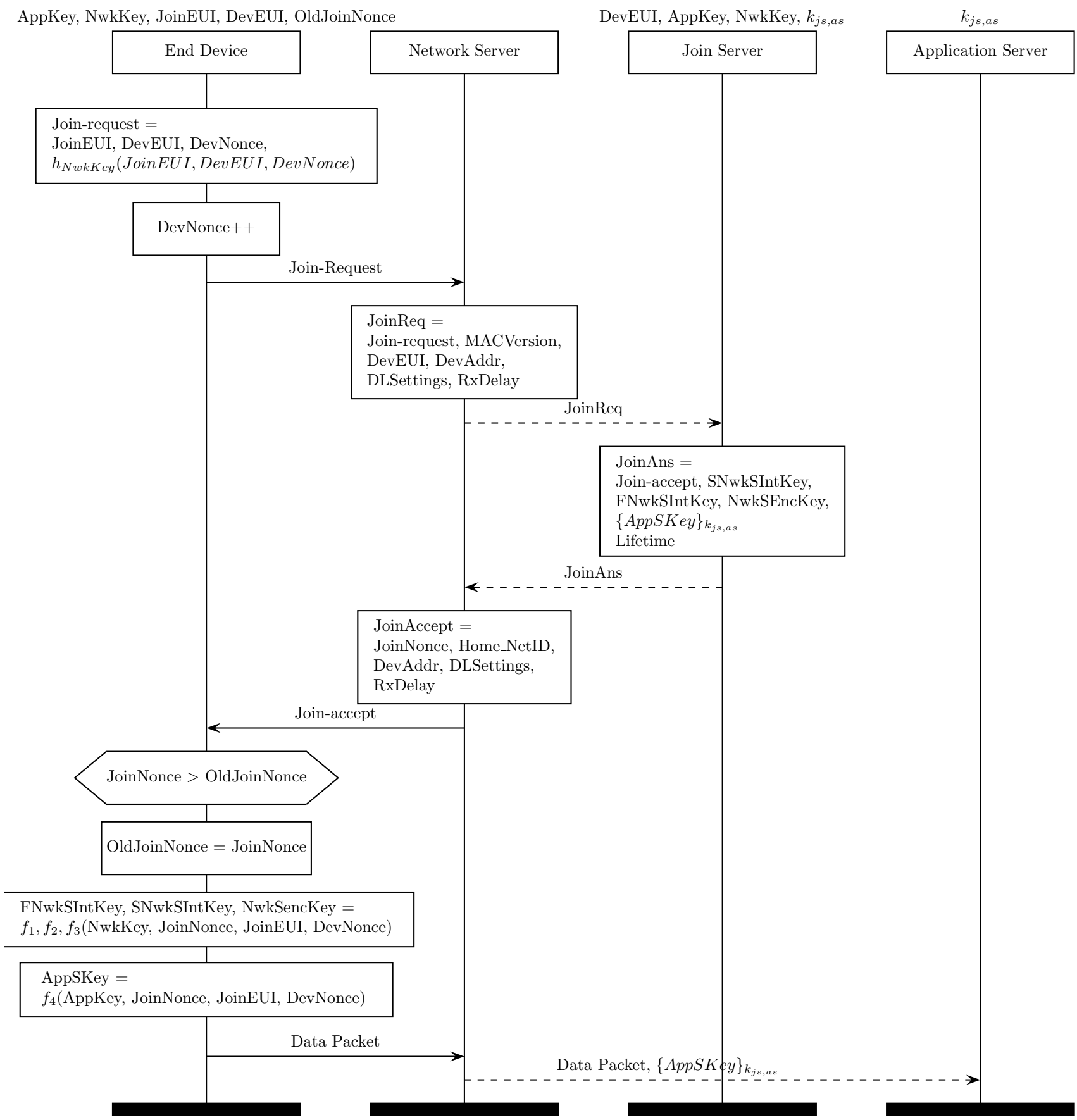

Figure 10: LoRa 1.1, AS Fetches AppSKey from NS 


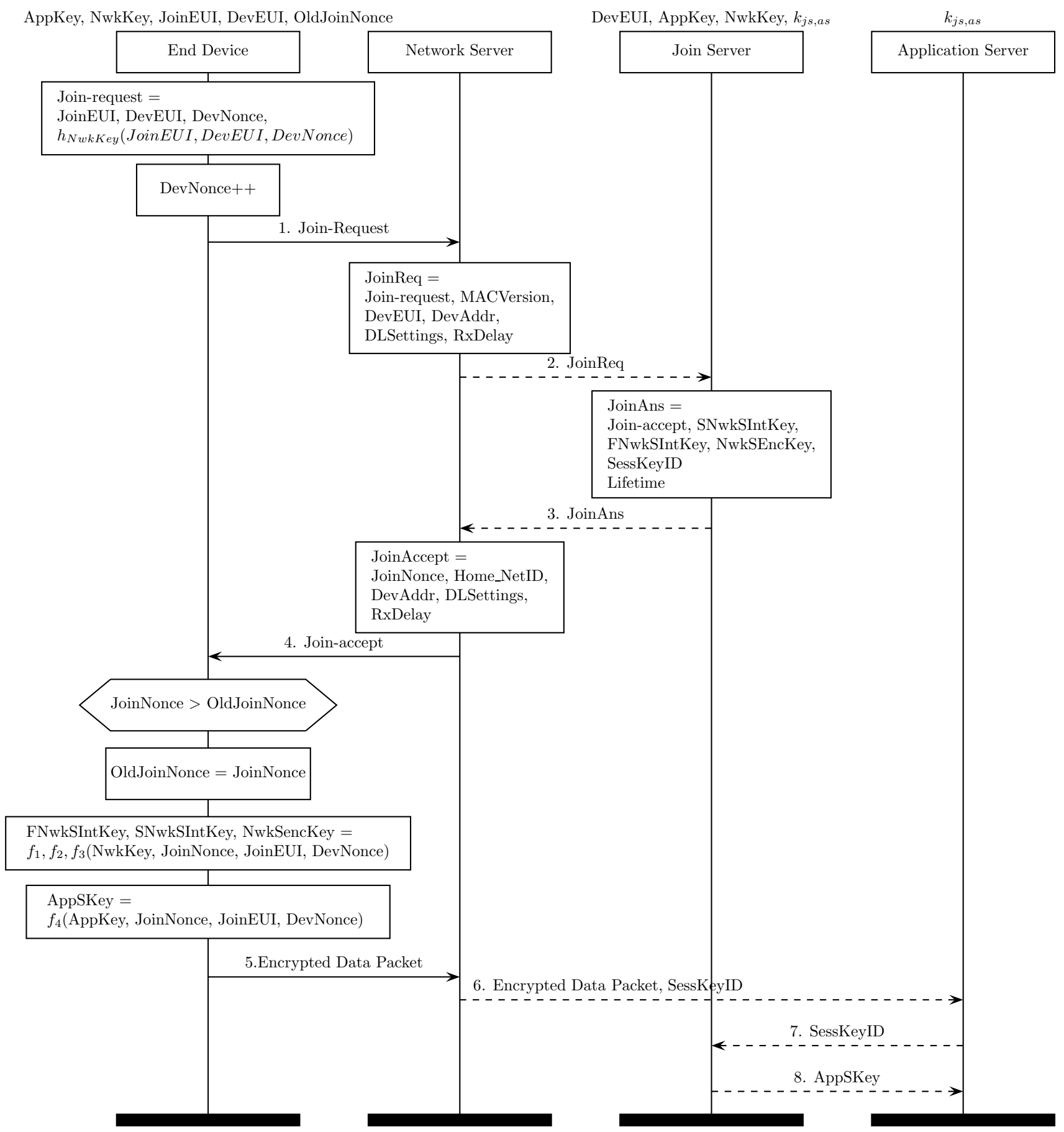

Figure 11: LoRa 1.1, AS Fetches AppSKey from JS 